\title{
Vers de nouveaux territoires d'intervention: émergence et usages de la simulation du travail à distance
}

Towards new territories of intervention: emergence and uses of remote

simulation of work

Laurent Van Belleghem

\section{(2) OpenEdition}

\section{Journals}

Édition électronique

URL : https://journals.openedition.org/activites/7083

DOI : 10.4000/activites.7083

ISSN : 1765-2723

Éditeur

ARPACT - Association Recherches et Pratiques sur les ACTivités

Référence électronique

Laurent Van Belleghem, «Vers de nouveaux territoires d'intervention : émergence et usages de la simulation du travail à distance », Activités [En ligne], 18-2 | 2021, mis en ligne le 15 octobre 2021, consulté le 07 avril 2022. URL : http://journals.openedition.org/activites/7083 ; DOI : https://doi.org/ 10.4000 /activites.7083

Ce document a été généré automatiquement le 7 avril 2022.

\section{(i) $(9)$}

Activités est mis à disposition selon les termes de la licence Creative Commons Attribution - Pas d'Utilisation Commerciale - Pas de Modification 4.0 International. 
Vers de nouveaux territoires d'intervention : émergence et usages de la simulation du travail à distance

\author{
Towards new territories of intervention: emergence and uses of remote \\ simulation of work
}

Laurent Van Belleghem

\title{
NOTE DE L'ÉDITEUR
}

Article soumis le 17/05/2021, accepté le 06/09/2021

\section{Introduction}

1 La crise sanitaire de la Covid-19 qui s'est déclarée en 2020 a affecté notablement les modalités de réalisation du travail pour une majorité d'entreprises et d'organismes, notamment à travers l'application de restrictions de différentes natures : confinement, couvre-feu, télétravail imposé, distanciation sociale, limitation des regroupements, jauge d'occupation des locaux, etc. Ces mesures, associées à l'exigence de continuité de l'activité économique, ont contribué dans un certain nombre de secteurs à des formes de mise à distance du travail, appuyées principalement sur l'usage de technologies numériques, notamment de visioconférence. Ces modalités nouvelles ont reconfiguré profondément les communautés de travail exerçant traditionnellement en présentiel, en transformant à la fois leur rapport au lieu géographique d'exercice du travail d'une part, leurs relations aux collègues et aux bénéficiaires de leur activité d'autre part, et jusqu'au contenu même de leur travail enfin, notamment par l'usage quasi-exclusif de l'outil informatique pour le réaliser. Ce sont dès lors les territoires du travail, c'est-à-dire 
les écosystèmes de relations structurant l'agir productif commun, qui se sont vu profondément bousculés et restructurés. Si certaines de ces transformations sont assurément réversibles, il est probable qu'un grand nombre d'entre elles s'inscrive de façon durable dans les pratiques d'entreprises et dans le paysage professionnel français.

2 Les ergonomes n'ont pas échappé à ces bouleversements, dont l'exercice du métier s'est vu triplement affecté par 1) l'application des restrictions sanitaires nationales valant tout autant pour nombre d'entre eux, 2) la transformation profonde de leur objet de travail qu'est le travail des autres et ses conditions de réalisation 3) la transformation des conditions d'accès à l'activité des travailleurs et des travailleuses, qu'il s'agisse d'en effectuer l'analyse par l'observation ou de contribuer à sa transformation par l'animation de collectifs d'acteurs en capacité de s'en saisir, à travers notamment la production et l'usage d'objets intermédiaires. Autrement dit, les territoires d'intervention des ergonomes se sont vus bousculés autant que les territoires du travail des bénéficiaires de leurs prestations se sont transformés. Ils ont dès lors intérêt à évoluer dans des directions permettant d'appréhender mieux les formes imposées par les réalités du travail d'aujourd'hui, et probablement de demain.

Cet article se propose de rendre compte comment de nouveaux territoires d'intervention de l'ergonomie ont pu être explorés par la mise en œuvre de ce geste professionnel d'ergonome qu'est celui de la simulation du travail dans un contexte où il ne pouvait être mené, comme à l'habitude, de façon présentielle.

4 Après avoir précisé la notion de territoire d'intervention et rappelé les exigences méthodologiques de la simulation du travail, trois interventions engagées sur l'année 2020 dans le contexte pandémique sont détaillées pour rendre compte de la naissance et de la consolidation de la simulation du travail à distance, que l'on peut aussi nommer simulation distancielle. Ces interventions ont permis d'investir, malgré la distance, trois domaines privilégiés de l'ergonomie: le conseil en prévention, la conception de situations de travail et la formation. Pour chacune d'elles sont précisées les modalités qui ont permis de mener, en tant que geste professionnel incarné, les simulations du travail, mais aussi comment elles ont été rendues possibles par le jalonnement d'un territoire d'intervention singulier permettant l'engagement large de l'écosystème d'acteurs des organismes demandeurs.

On notera que cette approche par le territoire (intégrant a priori nécessairement une dimension géographique) peut sembler contre-intuitive pour rendre compte d'activités réalisées à distance (c'est à dire supposées affranchies de la contingence géographique). Pour autant, le travail ne s'affranchit jamais totalement d'un ancrage local, ne serait-ce que parce que les travailleurs se trouvent toujours dans un lieu physique réel (qu'il s'agisse d'un bureau d'entreprise, d'un chez-soi réaménagé, d'un tiers-lieu providentiel, d'un espace de co-working, d'un véhicule de service...), mais aussi parce que le travail est le produit d'un agir collectif qui s'inscrit toujours dans une réalité spatio-temporelle qu'il nous faut savoir définir. Le propos retenu tente de rendre compte de cet apparent paradoxe tout en précisant, par précaution, qu'il s'agit ici d'une approche exploratoire. 


\section{Quand la crise sanitaire dessine de nouveaux territoires du travail}

\section{Des évolutions de la notion de territoire}

6 La notion de territoire est une notion mouvante, renvoyant à une diversité de significations selon la discipline, l'angle d'approche retenu ou l'époque de son usage. Dans le champ de l'économie politique, Blanc (2020) rappelle ainsi que le mot "territoire » a changé plusieurs fois de sens. Désignant, à la sortie de la Première Guerre mondiale, les frontières délimitant des systèmes de pouvoirs spécifiques (capacités militaires, découpages administratifs...), l'emploi du terme évolue vers «l'aménagement du territoire " (infrastructures, moyens économiques...) avant d'envisager plus spécifiquement les flux (de circulation, d'investissements...) traduisant ses caractéristiques économiques et/ou sociales. Aujourd'hui, et sans en faire disparaitre totalement le caractère géographique, le territoire se définit plus volontiers par les « sujets » qu'il réunit au sein d'un écosystème d'acteurs disposant de ressources, matérielles ou symboliques, pour agir collectivement. Demissy (2018) complète cette analyse en mettant en lien la modification des rapports des individus et des organisations à l'espace avec la mutation des modèles économiques. Dans une économie industrielle classique, où l'enjeu du travail vise la production d'un produit standardisé, le territoire se définit en rapport à un espace constitué de ressources principalement matérielles (matières premières, implantations, main d'œuvre disponible, infrastructures, transports...). Dans une économie servicielle telle qu'elle tend à se généraliser (Du Tertre, 2012), l'enjeu économique se joue désormais dans l'existence d'une relation de travail où le bénéficiaire est coproducteur du service et où la dimension immatérielle tend à devenir centrale. De fait, le territoire s'entend là encore d'abord comme un écosystème d'acteurs, fait d'interdépendances, de relations et d'actions collectives (Asselineau, Albert-Cromarias, \& Ditter, 2014; Blanc, 2020). En ergonomie, Boudra et Béguin (2013) définissent eux aussi le territoire comme «le résultat d'un processus d'interaction entre différents acteurs » (p. 2) de façon à comprendre comment les dynamiques reliant différents niveaux décisionnels (macro, méso, micro) structurent les conditions de travail locales (Boudra, 2016).

7 Cette orientation de la notion de territoire sur les interactions entre acteurs en ouvre une autre. Mesnard (2003), en suivant Di Méo (1998), retrace en effet la notion de territoire selon une double filiation, conduisant à deux conceptions extrêmes: le territoire politique et le territoire éthologique. Le territoire politique renvoie à la définition historique d'un espace sur lequel s'exerce une autorité, articulant entre elles une aire géographique, un régime de pouvoir et une organisation sociale. Le territoire éthologique, de définition plus récente, désigne l'espace occupé par un animal et qu'il défend comme sa propriété exclusive vis-à-vis d'autres animaux, y compris de la même espèce. Par métaphore, ce processus d'appropriation "naturelle » s'apparente à une appropriation "culturelle et sociale » propre à l'humain. Mesnard (2003, p. 66) voit dans la combinaison de ces approches politique et éthologique un processus de territorialisation articulant une action d'aménagement du territoire pour satisfaire les besoins du groupe à une action d'appropriation d'un territoire qu'il s'agit de conquérir et de délimiter. Autrement dit, le territoire n'est pas seulement un lieu aménagé (politiquement) pour que des activités s'y déploient. Il est aussi le produit des activités 
de ceux qui l'explorent («éthologiquement »), le jalonnent et en définissent les règles et les valeurs ${ }^{1}$. Dans ce double mouvement d'aménagement et d'appropriation, et suivant ici Le Berre (1992), Mesnard repère trois facettes au territoire: une facette physique (constituée des configurations territoriales naturelles ou artefactuelles), une facette organisationnelle (constituée du système de règles définissant les relations entre les acteurs sociaux) et une facette existentielle (dotant le territoire d'une identité propre, résultat du processus d'appropriation).

Dans le champ du travail, on peut ainsi comprendre les territoires du travail comme des écosystèmes d'acteurs engagés dans un agir productif commun (caractérisé notamment par des enjeux de coopération), à la fois aménagés pour agir et résultats de cet agir commun. On pourra en repérer les dimensions physique (constitué des lieux d'exercice du travail favorisant la proximité physique des travailleurs et mettant à leur disposition les ressources matérielles et immatérielles utiles à l'exercice de leur activité), organisationnelle (constitué du corps de règles formelles et informelles prescrivant l'agir productif commun) et existentielle (porté notamment par l'histoire, les raisons d'être et les valeurs des entreprises et des populations y œuvrant).

Mais que deviennent ces écosystèmes d'acteurs s'ils se voient soudain privés (à l'occasion d'un confinement national par exemple) de la proximité physique sur laquelle s'appuyaient jusqu'alors ces coopérations ? Et que deviennent les territoires du travail si, par le recours à des technologies nouvelles, de type visioconférence notamment, ces écosystèmes d'acteurs s'organisent pour faire perdurer, malgré l'éloignement imposé, les interactions leur permettant d'agir et de produire collectivement? Faut-il voir ici une disparition des territoires du travail avec la disparition d'une de leurs dimensions ? Ou au contraire faut-il y voir l'émergence d'une autre nature de territoire, dont la facette physique intègre à son profit les nouvelles technologies pour reconstituer un ancrage géographique « à distance » afin de garantir la continuité de l'activité et l'identité de son écosystème d'acteurs ?

Pour le dire autrement, quels nouveaux territoires le travail à distance dessine-t-il ?

\section{Des écosystèmes d'acteurs redéfinis par le travail à distance}

11 De fait, les territoires du travail semblent s'affranchir un peu plus, dans cette parenthèse pandémique, de leur ancrage à un lieu géographique donné. Ainsi, la diffusion massive et rapide du télétravail, qui a prolongé le travail à domicile imposé par le premier confinement de mars 2020, a bousculé le lien du salarié à son entreprise, poussant certains ménages à changer de région sans changer d'emploi pour gagner en qualité de vie. Cette tendance n'a pas échappé aux partenaires sociaux. L'accord national interprofessionnel négocié cette année-là (ANI, 2020), s'il appelle d'abord à garantir la cohésion sociale dans cette période particulière, invite aussi à accompagner des formes de travail pouvant répondre aux demandes de certains salariés et de certaines entreprises, notamment dans la possibilité d'élargir l'accès au télétravail au regard de ses bénéfices souvent constatés : réduction des déplacements, autonomie, équilibre vie privée-professionnelle, attractivité de l'entreprise, fidélisation des salariés... De fait, c'est l'ensemble de la répartition géographique de la main d'œuvre sur le territoire national qui se voit ainsi potentiellement mis en mouvement par l'extension massive du télétravail. Il y a là une forme inédite d'extension des territoires du travail. 

Si l'ANI (2020) précise que les activités éligibles au télétravail doivent être "télétravaillables", rien n'est dit sur la transformation qu'opère la réalisation à distance d'une activité traditionnellement réalisée en présentiel. Qu'en est-il, notamment, de la contribution de l'activité « télétravaillée » à l'agir productif commun de l'écosystème d'acteurs de l'entreprise? Quelle forme cette activité prend-elle quand il s'agit d'assurer un service dont on a rappelé qu'il se réalisait dans le cadre d'une coproduction entre le (télé)travailleur et le (télé)bénéficiaire? Quelles transformations des gestes professionnels des travailleurs, parfois élaborés de longue date dans un cadre strictement présentiel, le truchement des outils numériques impose-t-il ? Et comment l'ergonome, dont l'action porte précisément sur ces écosystèmes d'acteurs pour, avec eux, «transformer le travail », réinvestit-il ses gestes professionnels dans une intervention à distance?

\section{Vers une offre d'ergonomie à distance}

13 L'ergonomie est-elle une activité télétravaillable ? La question mérite d'être posée tant le premier confinement de mars 2020 a tétanisé la profession sur ce point. En effet, comment analyser le travail et contribuer à sa transformation quand l'accès à l'activité se voit soudain fermé ? L'usage de la visioconférence notamment, désormais installé dans les pratiques des entreprises et des travailleurs, suffit-il aux ergonomes pour intervenir sur le travail?

Des initiatives comme celle des "Ergonomes Solidaires" ont tenté, très vite, de présenter une offre de service à distance en proposant un service téléphonique d'écoute et de conseil de premier niveau sur l'installation des postes de ces nouveaux «travailleurs à domicile» et sur la reprise du travail en entreprise (Mazzola \& Allain, 2020 ; Zittel, 2020). Pour autant, personne n'ignorait que cette offre était très éloignée des pratiques traditionnelles de l'ergonomie, voire en contredisait les principes conceptuels en s'affranchissant de l'observation du travail et en proposant un conseil de type expert. La page d'accueil du site internet créé pour l'occasion alertait d'ailleurs sur le fait que cette offre ne saurait se substituer à une "intervention ergonomique de terrain ».

Est-ce à dire que l'impossibilité d'accéder physiquement «au terrain » empêche définitivement la réalisation d'une intervention ergonomique? Le territoire d'intervention des ergonomes serait-il ici plus attaché au lieu géographique de réalisation du travail qu'à l'écosystème d'acteurs œuvrant dans le cadre d'un agir collectif ? Rien n'est moins sûr. Il y avait lieu, dès lors, d'envisager la mise en œuvre de nos gestes professionnels d'ergonomes dans un mode distanciel qui ne trahisse pas les principes conceptuels de l'ergonomie. C'est ici un enjeu de développement du métier, dont on sait qu'il ne s'appuie pas uniquement sur la mobilisation de connaissances abstraites, mais aussi sur des expériences humaines incarnées dans des gestes professionnels dont la dimension corporelle est centrale (Gal-Petifaux \& Roche, 2015), et ce que quelles que soient les pratiques sociales concernées (enseignement, médecine, coaching sportif, conseil... intervention ergonomique).

C'est dans ce cadre que nous avons proposé un dispositif opérant permettant la mise en œuvre à distance d'un geste professionnel important en ergonomie : la simulation du travail. Si celui-ci est structuré par un cadre prescriptif de ce qu'il devrait être (cf. 
infra), il se déploie aussi comme une expérience sensible engageant autant le corps que l'esprit de celui qui l'exécute. La façon dont l'intervenant, notamment, anime et donne vie à un dispositif tangible qu'il a préalablement imaginé pour accompagner des participants dans un processus de simulation invitant lui aussi à une activité corporelle, via la manipulation d'objets tangibles, relève d'un geste professionnel intégrant de façon complexe une multiplicité de dimensions (Gal-Petifaux \& Roche, 2015) : motrice (gestuelles, déplacements, postures), perceptive (sensoriel, kinesthésique), émotionnelle (ressentis, sentiments), langagière (phonétique, linguistique), sémiotique (intentionnalités, connaissances, valeurs, croyances, raisonnements).

De ce fait, et au-delà du dispositif de simulation proprement dit, l'organisation des ateliers de simulation fait traditionnellement l'objet d'une préparation minutieuse dans le choix des lieux et de leur configuration spatiale et matérielle, dans la définition des consignes et des règles d'interaction à tenir au cours des simulations, ainsi que dans le choix du nombre et du profil des participants pour favoriser au mieux la mise en œuvre de ce geste professionnel. Ces éléments liés à l'organisation des ateliers sont par ailleurs intégrés à un dispositif d'intervention plus large mettant sur pied plusieurs instances (comité de pilotage, comité de suivi...) elles-mêmes mises en lien avec les acteurs du projet (chefferie de projet, bureau d'études, architectes...) ainsi qu'avec les instances décisionnelles et fonctionnelles des organismes concernés (Codir, Comex, CSE, DRH, etc.) vis-à-vis desquelles les modalités de restitution, d'arbitrage et d'accompagnement des prises de décision devront aussi être définies. Autrement dit, les intervenants, en intégrant le territoire du travail de l'entreprise (entendu comme un écosystème d'acteurs singulier avec lequel il s'agit d'interagir), marquent les jalons de leur propre territoire d'intervention en délimitant le champ, les acteurs, et les modalités physiques et organisationnelles des interactions qu'ils auront à y mener. C'est le balisage de ce territoire d'intervention, dans ses facettes physique, organisationnelle et existentielle, qui doit permettre, entre autres, la mise en œuvre efficace du geste professionnel qu'est la simulation du travail dans toutes ses dimensions et d'en tirer les enseignements pour l'instruction du projet.

Dès lors, l'enjeu visant à inventer un dispositif socio-technique de simulation à distance se devait d'être attentif à deux objets: la définition d'un territoire d'intervention affranchi de ses repères géographiques traditionnels (lieux de travail, salles de réunion, lieux de pause - et leurs précieuses régulations informelles...) et la façon dont les multiples dimensions du geste professionnel allaient pouvoir être ré-investies dans l'expérience vécue des intervenants et des participants sans le support habituel de la proximité physique.

\section{La simulation du travail à distance : principes}

\section{La simulation du travail : la nécessité d'un dispositif tangible?}

19 La simulation du travail est une méthodologie venant outiller les démarches de conception ergonomique des situations de travail (Van Belleghem, 2018a) faisant l'objet, ces dernières années, d'un développement actif dans l'exploration de nouveaux champs d'intervention et de questionnements en France (Besnard \& Levy, 2017; Bobillier Chaumon, Rouat, Laneyrie, \& Cuvellier, 2018; Casse, 2015 ; Cheyrouze 2021 ; Haradji, 2021; Heddad, 2019; Ughetto, 2018; Van Belleghem, 2012; Vispi, Lebru, 
Lepreux, Chaabane, \& Kolski, 2021) comme à l'international (Andersen \& Broberg, 2016 ; Braatz, Paravizo, \& Fontes, 2019 ; Conceição \& Broberg, 2019).

Le principe général de la méthodologie vise à soumettre le projet de transformation d'une situation de travail à l'épreuve des logiques d'action des travailleurs concernés et, ce faisant, d'engager un processus d'instruction itératif ouvrant la perspective d'une meilleure adaptation du projet à l'activité (Figure 1a).

Un dispositif socio-technique est spécifiquement mis en œuvre dans cet objectif (Figure 1b). Il regroupe généralement un collectif d'acteurs porteurs d'exigences propres quant au projet en cours (concepteurs et prescripteurs, travailleurs concernés, encadrement, décideurs, représentants du personnel, etc.) autour d'un support spécifique (une maquette, le plus souvent) permettant d'instruire le projet en cours en élaborant des scénarios de prescription avant de les soumettre à la simulation du travail. Cette dernière vise à proposer aux travailleurs présents de «jouer» leur activité future sur le support à l'aide d'avatars (Van Belleghem, 2012) et d'évaluer ainsi l'adéquation du projet à leurs propres logiques d'action, éventuellement en faisant évoluer ces dernières. L'engagement d'un processus alternant scénarisation et simulation contribue ainsi à l'exploration, puis à la sélection, de scénarios susceptibles de répondre conjointement aux enjeux des travailleurs et de l'entreprise jusqu'à la consolidation d'un scénario privilégié. À l'issue de la démarche, le scénario retenu ainsi que les critères ayant conduit à son élaboration font l'objet d'une formalisation par l'intervenant ergonome, traditionnellement sous la forme d'un cahier des charges ergonomiques venant nourrir et structurer le projet.

Figure 1 : Modèles de la situation de simulation et du dispositif socio-technique associé Figure 1 : Models of the simulation situation and the associated socio-technical device
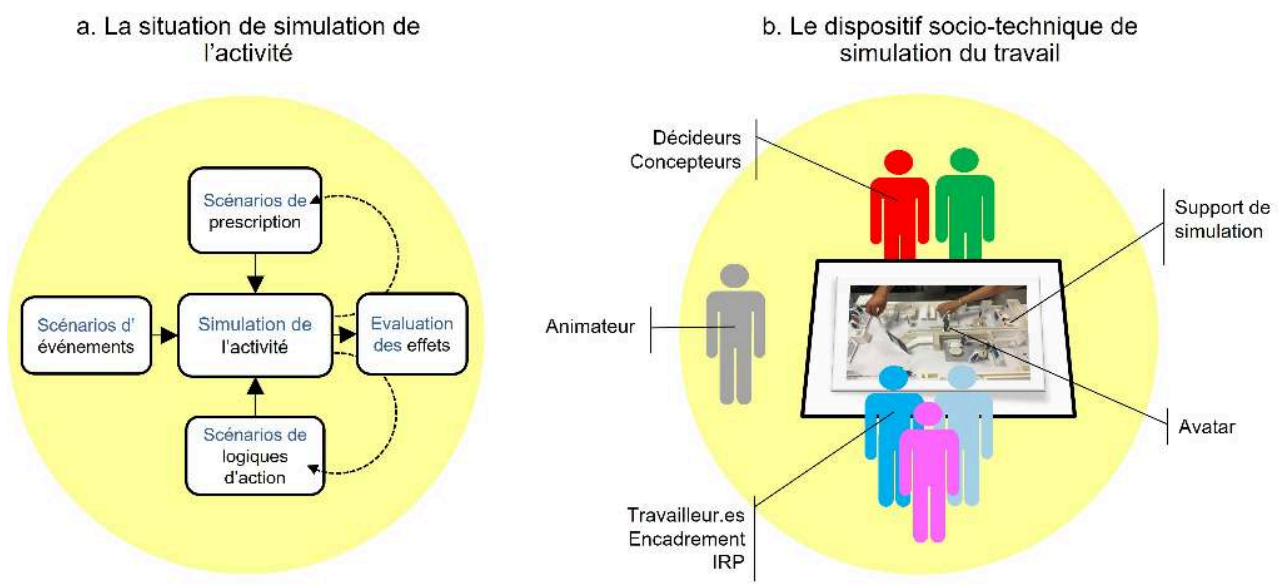

Ce dispositif socio-technique, relativement complexe, requiert le regroupement physique des acteurs dans un même lieu et le recours à un matériel tangible dont la manipulation par les participants est un principe opératoire de la démarche : chacun doit pouvoir faire des propositions sur les supports à disposition (déplacer une cloison ou transformer un espace sur une maquette architecturale, proposer une nouvelle fonction ou rattacher un service à un autre sur une maquette organisationnelle...), imaginer et produire de nouvelles situations à simuler (renseigner des cartes «situation », structurer un scénario temporel d'événements à faire jouer...) et, surtout, saisir et manipuler les avatars mis à disposition pour simuler le travail futur. 
Ce principe de saisie de l'avatar est particulièrement puissant dans le mécanisme de projection dans l'activité future, dont l'usage du pronom personnel «je» suivi d'un verbe d'action conjugué ( $j$ 'arrive sur mon lieu de travail... je passe par le vestiaire... je vais chercher les consignes... ») est un marqueur. En effet, si la verbalisation de l'activité accompagne l'action simulée grâce à l'avatar, elle ne la précède pas. C'est l'avatar, par sa capacité d'incarner l'activité, qui permet la simulation cognitive de l'activité et, ce faisant, sa mise en mots (Van Belleghem, 2018a). Dès lors, la mise à disposition d'un avatar tangible à manipuler sur un support lui-même tangible s'avère centrale, voire incontournable, dans la mise en place d'un dispositif socio-technique de simulation du travail, y compris pour des projets de nature plus abstraite comme des projets organisationnels ou digitaux.

列 même de l'impossibilité apparente de proposer des supports et avatars tangibles à la manipulation de participants regroupés, par exemple, en visioconférence ? Pas si les outils numériques mis à disposition à cette occasion permettent d'engager un rapport tangible à des objets produits et manipulés collectivement, y compris à distance. C'est ce dispositif socio-technique que l'ergonome doit savoir concevoir pour co-produire, avec l'écosystème d'acteurs qu'il réunit, un nouvel agir commun.

\section{Vers un dispositif de simulation du travail à distance}

Le dispositif numérique de simulation du travail à distance doit, tout d'abord, permettre aux participants d'interagir entre eux. Des outils de visioconférence de type Zoom, Teams, Jitsi Meet, BigBlueButton... sont des outils désormais répandus et offrant régulièrement de nouvelles facilités d'usages et d'interaction.

Le dispositif doit ensuite permettre la visualisation et la manipulation d'un support de simulation ad'hoc. Trois exigences sont requises pour concevoir un support de simulation tangible utilisable en présentiel (Van Belleghem, 2012), et le sont de fait tout autant pour un support en distanciel :

- Exigence de représentation: le support doit permettre de représenter le système de prescription en cours de conception sous forme de scénarios. Les outils de visioconférence permettant aujourd'hui presque tous de partager l'écran de l'animateur ou de l'un ou l'autre des participants, donner à voir une représentation conçue sur une application quelconque (un plan 2D sous Autocad, un plan 3D sous Sketchup ou encore une schématisation sous PowerPoint) ne présente plus aucune difficulté. Cette exigence est donc parfaitement tenable.

- Exigence de modification: le support doit permettre à chaque participant de modifier la représentation du système de prescription en cours de conception et contribuer ainsi au travail de scénarisation du projet. Centrale dans une démarche participative s'appuyant sur la capacité de production collective des participants, cette exigence est assurément la plus difficile à garantir à distance. Les outils numériques couvrent en effet classiquement deux grands types d'usages en distanciel: le travail de production individuelle (via des outils bureautiques de type Word ou Excel par exemple) et la tenue de réunions de travail centrées sur des interactions verbales (via des outils de télé ou de visioconférence). Les outils de production collective, permettant d'agir à plusieurs sur un même document, sont encore rares ou d'usage plus spécialisé dans le monde du travail'2. Pour autant, des solutions comme Sharepoint permettent aujourd'hui de travailler à plusieurs sur un même document, de type 
ppt ou word par exemple. De la même façon, de nouveaux outils collaboratifs, comme Klaxoon ou Miro, ciblent clairement le marché du travail d'équipes distantes par la mise à disposition de «tableaux blancs » (whiteboard) venant soutenir la réflexion et la production collective. Ces outils peuvent fournir des pistes fécondes pour outiller la scénarisation collective.

- Exigence de simulation: le support doit permettre d'y déplacer des avatars, ceux-ci devant être "préhensibles » par les participants invités à venir jouer leur activité. Sans viser des interactions très sophistiquées (comme pourraient l'être des mouvements complexes de personnages virtuels via des manettes de jeux à boutons multiples), on cherchera cependant à permettre la saisie d'objets virtuels représentés à l'écran via le pointeur de souris du participant et lui permettre de les déplacer sur le support représenté sur l'écran partagé. «Donner la main» est ici une fonction centrale, mais encore peu fréquente sur les outils classiques de partage d'écran. Teams permet ainsi, selon certaines configurations, de «donner le contrôle » à un participant en faisant apparaître son pointeur de souris sur l'écran en plus du pointeur de l'animateur. Cette fonction simple ouvre immédiatement des possibilités de manipulation d'objets à distance qu'il est possible d'exploiter dans un objectif de simulation. D'autres outils comme Miro ou Mural, conçus pour la production collective et faisant volontairement apparaître l'ensemble des pointeurs de souris et l'identification de leur utilisateur sur le board partagé, seront d'autant plus adaptés à l'enjeu de simulation à distance qu'ils pourront aussi permettre des simulations à plusieurs et avec une grande fluidité.

La réunion de ces exigences doit ainsi permettre de constituer un dispositif distanciel de simulation $d u$ travail, permettant de faire interagir, scénariser et simuler un écosystème d'acteurs, quelle que soit l'implantation géographique de chacun d'eux pris isolément (Figure 2).

Figure 2 : Dispositif distanciel de simulation du travail. Figure 2 : Remote work simulation device

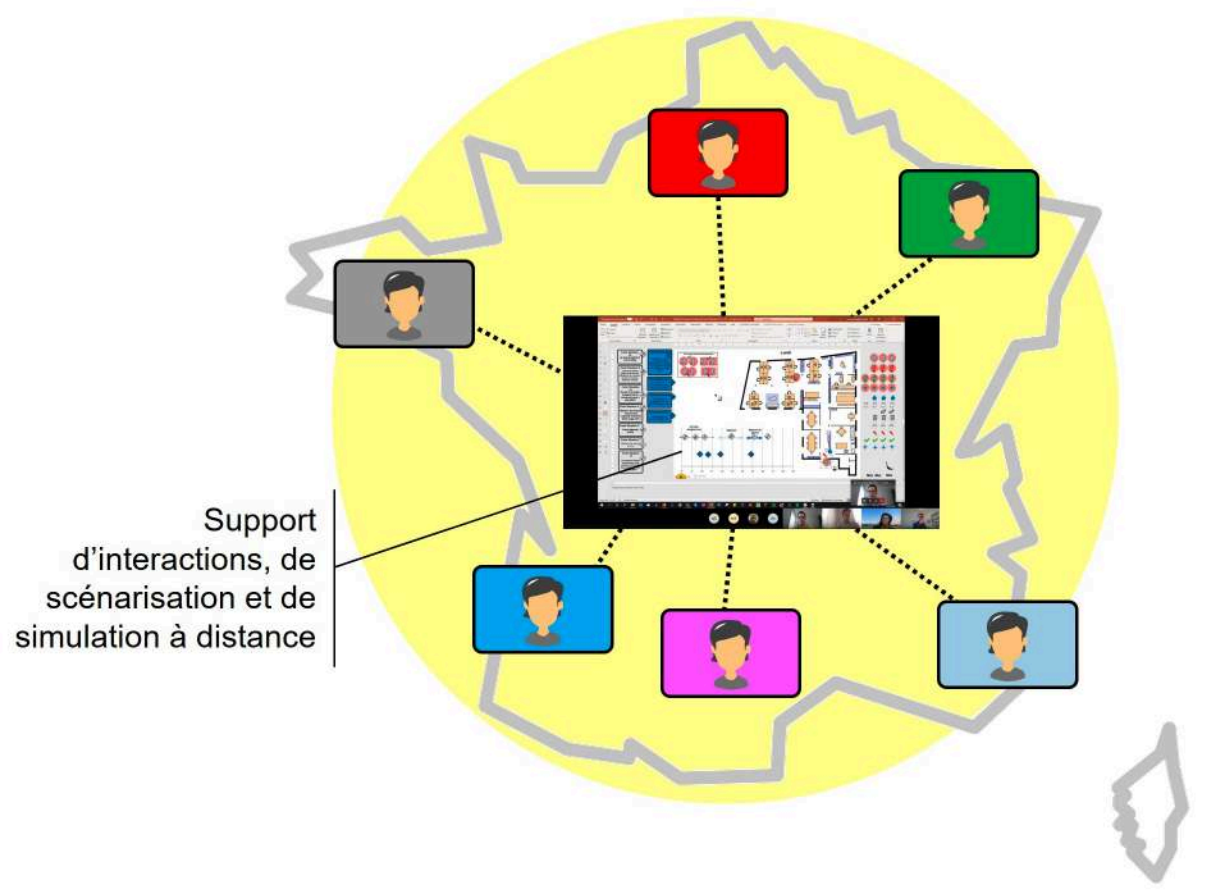


L'intention et les moyens du dispositif socio-technique étant posés, l'animation des simulations peut commencer.

\section{Nouveaux territoires de la prévention : simuler le risque que fait courir le retour au présentiel}

\section{Un cabinet-conseil face à la perspective du déconfinement}

29 À la fois bureau d'études et cabinet de conseil, Plein Sens ${ }^{3}$ est spécialiste des questions sociales et du design des organisations. Comme la majorité des salariés en France, sa trentaine de consultants se voit confinée en mars 2020. Plusieurs d'entre eux font le choix de se confiner en province, loin des locaux parisiens de l'entreprise, pour y trouver un environnement de travail et de vie le plus favorable possible. Très rapidement, la direction du cabinet met en place un dispositif, dit « Woodstock », visant à reconduire en distanciel tout le fonctionnement de l'équipe précédemment réalisé en présentiel. Les rituels sociaux (réunions d'équipe, comités de direction, comités de production, réunions des mandataires sociaux, etc.) sont tous replanifiés en visioconférence. Ils sont complétés d'un dispositif de communication permettant à l'ensemble de l'équipe, à travers une diversité d'outils partagés, de maintenir une dynamique de réflexion collective autour de problématiques communes, ainsi que d'un groupe Whatsapp pour échanger plus librement et légèrement sur le confinement. L'enjeu de continuité de l'activité économique, en lien avec des clients eux-mêmes contraints au fonctionnement distanciel, prend ainsi appui sur cette nouvelle structuration interne de l'écosystème d'acteurs du cabinet.

30 En avril 2020, et après deux reconductions, la sortie du confinement se précise pour le 11 mai. Pour Plein Sens, comme pour toutes les entreprises, l'échéance n'est pas anecdotique : il s'agit de préparer le retour des salariés dans leurs locaux en intégrant des mesures de prévention inédites face à une menace sanitaire elle-même inédite, que chacun sait potentiellement mortelle. Des prescriptions légales et réglementaires génériques (date de déconfinement, principes de distanciation sociale, port d'équipements de protection individuelle...) sont déclinées en prescriptions sectorielles diffusées par le Ministère du Travail (Fiches-conseils métiers) que les entreprises doivent transformer en prescriptions locales sur plusieurs domaines possibles: obligations de port et mise à disposition de moyens de protection (masques, gants, gel hydroalcoolique...), prescriptions liées aux usages des espaces de travail (jauge d'occupation des locaux, règles de circulation, obligation de distanciation physique...), changements éventuels d'horaires de travail et d'ouverture des locaux pour limiter les croisements de personnels, etc.

31 Cette cascade de mesures de prescription, allant directement du sommet de l'État à la situation de travail la plus singulière, ne dit cependant rien du «travail réel ». Quelle sera en effet la nature de l'activité de chaque salarié, le 11 mai, faisant face à ce nouveau cadre prescriptif? Quel risque une reprise du travail dans des conditions différentes « d'avant », mais peu instruites du point de vue de l'activité qui en émanera, fait-elle courir aux salariés? Quelles régulations les équipes vont-elles devoir opérer pour ajuster leurs logiques d'action collectives aux nouvelles règles de prévention et d'organisation? Et quels risques supplémentaires ce travail d'ajustement ajoute-t-il au risque de contamination déjà présent? 
'est pour ne pas laisser ces questions sans réponses qu'un dispositif de simulation de la reprise d'activité des consultants de Plein Sens dans leurs locaux est envisagé. L'exigence de mener cette réflexion avant le 11 mai combinée à l'impossibilité de pouvoir se réunir en présentiel invite à imaginer un premier dispositif de simulation à distance ${ }^{4}$.

\section{Un premier dispositif de simulation à distance}

Le dispositif imaginé s'appuie sur un système de visioconférence (ici la plateforme collaborative Microsoft Teams) offrant la possibilité à l'animateur de partager un document à l'écran avec l'ensemble des participants et de "donner le contrôle » à l'un d'entre eux pour lui permettre de manipuler les avatars et autres objets qui auront été mis à sa disposition. Le système fait apparaître sur l'écran partagé les pointeurs de souris de l'animateur et du participant ayant demandé le contrôle. C'est à ce dernier qu'il sera demandé de simuler son activité en déplaçant son avatar.

Le système permet ainsi à chaque participant de distinguer à l'écran trois zones enchâssées :

- la fenêtre du système de visioconférence interactif regroupant l'ensemble des participants autour d'un document partagé,

- la fenêtre partagée de l'application PowerPoint en mode conception (et non en mode diaporama) réunissant, à l'image d'une «table de jeu ", les différents éléments utiles à la simulation autour d'un " plateau de jeu »,

- un «plateau de jeu» central sur lequel les participants sont invités à venir simuler leur activité en venant déplacer leur avatar dans le plan des locaux.

Figure 3 : Structuration du dispositif de simulation utilisé.

Figure 3 : Structuring of the simulation device used

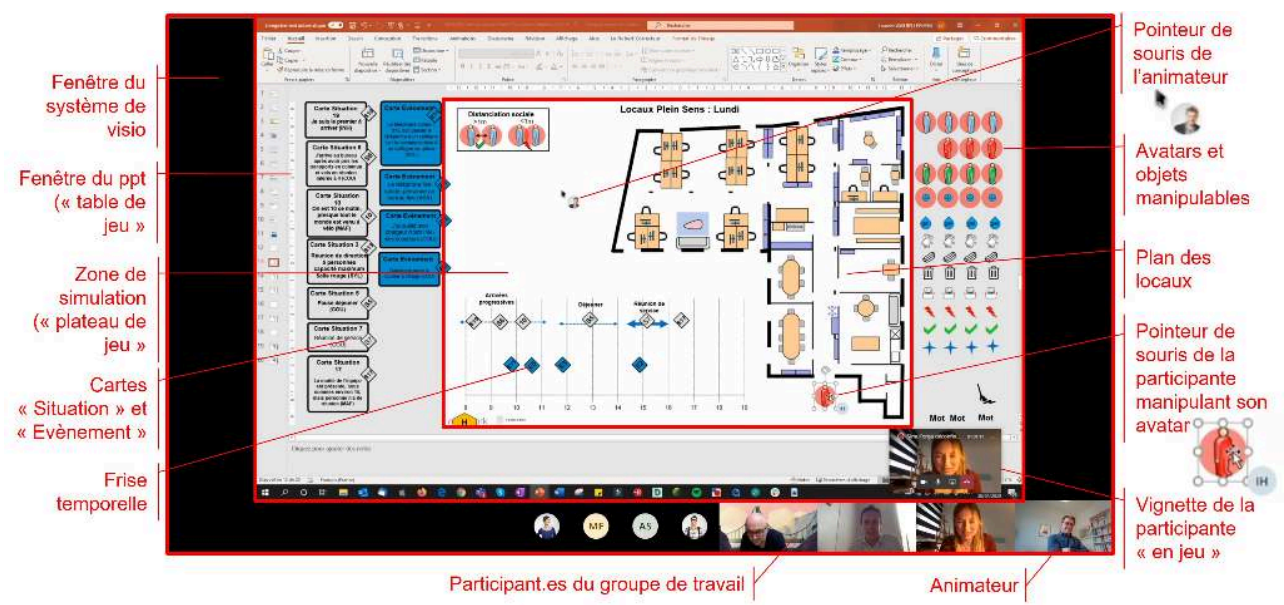

La «table de jeu » comprend de part et d'autre de la zone centrale de simulation :

- des cartes «Situation» et des cartes «Événement» numérotées, renseignées par les participants en amont des séances de simulation. Elles permettent de construire des scénarios d'événements en les positionnant chronologiquement sur la frise temporelle apparaissant dans la zone de simulation,

- Des avatars manipulables par les participants lors des simulations. Ils sont ici représentés avec une «zone de distanciation physique » autour d'eux permettant d'évaluer, lors des 
simulations, les distances minimales entre les salariés, facilitant ainsi l'évaluation de l'occupation maximale des différents espaces,

- Des objets du travail manipulables selon les besoins de la simulation. On y retrouve les moyens de protection sanitaire (gel hydroalcoolique, masque, etc.), mais aussi des objets et symboles potentiellement utiles lors des simulations. auront lieu durant la séance. L'objectif méthodologique, annoncé aux participants, est de pouvoir garder la trace des simulations de l'activité et des interactions qui l'accompagnent. À travers celles-ci, on peut en effet observer la construction progressive et collective d'une représentation partagée entre les participants de la réalité de l'activité à venir, leur permettant d'interroger les prescriptions envisagées initialement, les conduisant à les conforter, les préciser ou les modifier le cas échéant. Ces échanges filmés feront l'objet d'un montage a posteriori par les intervenants sur des séquences significatives de ce processus. Elles auront vocation à être présentées lors des restitutions ultérieures pour rendre compte aux parties prenantes et aux salariés du processus d'instruction tel qu'il a été appuyé par la simulation. Les extraits ci-après sont issus de ces séquences montées et restituées.

\section{Des simulations à l'origine d'une redéfinition du protocole sanitaire}

La séance vise à dérouler un scénario d'événements construit à partir des cartes Situation et Évènement rédigées par les participants en amont de la séance. Ce scénario reconstruit une journée de travail alternant des situations de différentes natures (arrivées progressives dans les locaux générant des occupations variables, travail de production individuel, réunion de direction, pause déjeuner, réunion de service...) émaillées d'évènements divers (appel téléphonique nécessitant de passer le téléphone à un collègue, apport du courrier par la gardienne...). Le choix des situations opéré par les participants rend compte de la peur légitime de la contamination, y compris dans les situations les plus communes ou les gestes apparemment les plus anodins. Les toutes premières simulations portant sur l'accès aux locaux, que l'échange ci-dessous retrace, en sont significatives.

Animateur: «première personne qui arrive... eh bien Inès ${ }^{5}$, allez... tu demandes l'autorisation... [Inès active la fonction de demande de contrôle, l'animateur y répond en activant le pointeur d'Inès] vas-y...

Inès : Alors, moi j'arrive... [saisit un des avatars sur l'écran] déjà, moi j'arrive en vélo... je gare mon vélo dehors... (...) et du coup je n'ai pas de masque sur mon vélo... euh, je ne sais pas en fait... a priori comme ça, je dirais, je n'ai pas mis de masque sur mon vélo... Comme j'ai touché les trucs et tout, j'ai les mains sales, au passage j'ai transpiré, enfin, je ne me sens pas de mettre un masque tout de suite en arrivant sans me laver les mains (...) bon bref, j'arrive devant la porte... [place son avatar devant la porte des locaux sur le plan] je cherche mes clés... j'ouvre... est-ce que je me suis lavé les mains avant?

Sylvie : non, tu pourras te laver les mains que quand tu seras à l'intérieur..

Claudy : ou alors il faut mettre à l'extérieur à disposition déjà du papier et de quoi

Activités, 18-2 | 2021 
s'essuyer les mains...

Matthieu: moi je pense que non... moi je pense que tu rentres et que tu te laves les mains une fois que tu es rentrée (...)

Claudy : mais après c'est vrai que la porte, très concrètement, moi je crois que je l'ouvre en poussant avec la clé déjà dedans, mais je ne la touche pas en fait...

Sylvie : oui, oui, je suis d'accord...

Matthieu : oui, moi aussi... »

L'analyse de cet échange et des actions associées (indiquées entre crochets) laisse tout d'abord apparaitre que le processus de simulation est à l'œuvre malgré l'usage d'un dispositif à distance. La participante invitée à simuler son activité est en capacité de saisir l'avatar la représentant et de le déplacer sur le plan pour en rendre visible les déplacements sur l'écran des autres participants en même temps qu'elle décrit son activité grâce à des verbes conjugués à la première personne du singulier ( $j$ j'arrive devant la porte... [place son avatar devant la porte des locaux sur le plan] je cherche mes clés... j'ouvre »). Le mécanisme de projection est à l'œuvre ! Il y a bien incarnation de l'activité à travers l'avatar et chaque participant peut en être spectateur, voire s'y projeter lui-même.

Le grain de l'activité ici simulée est par ailleurs révélateur de la problématique posée par le risque perçu de contamination cutanée. Il s'agit ici autant d'éviter de se contaminer en touchant les éléments de l'environnement que de les contaminer soimême. Le passage de la porte d'entrée devient dès lors un point particulièrement sensible dans le cours de l'action consistant à accéder aux locaux. La simulation de l'ouverture de la porte dans ce nouveau contexte s'appuie notamment sur la remémoration de la gestuelle singulière d'ouverture de cette porte chez plusieurs participants, se rappelant au passage qu'elle n'a pas de poignée et que l'ouverture se réalise avec la clé (« la porte, très concrètement, moi je crois que je l'ouvre en poussant avec la clé déjà dedans, mais je ne la touche pas en fait »). Il y a là une intrication féconde entre l'explicitation de l'action vécue passée (Vermersch, 1994) et la projection dans l'action future. C'est d'ailleurs ce retour vers le vécu que les participants exploitent ici pour débloquer la situation et autoriser l'ouverture de la porte sans nettoyage préalable des mains au motif que la porte ne fait pas l'objet de contact cutané.

41 Ce point instruit, la simulation se poursuit à l'intérieur des locaux.

"Inès [déplaçant son avatar à l'intérieur des locaux] : « du coup je vais me poser... par exemple aujourd'hui je vais m'installer ici... [déplace son avatar jusqu'à un poste de travail] j'ai fait tout le tour de l'open-space au passage

Claudy : et quand est-ce que tu as pris un masque dans tout ça?

Inès : ben, j'en ai pas pris du tout... euh, soit j'ai un masque avec moi dans mon sac... soit il y en a... je ne sais pas où... (...)

Sylvie : normalement, d'après ce que j'avais imaginé (...) c'était que chacun ait un stock à lui dans son casier... [Inès saisit un symbole "masque" et le dépose dans la zone des casiers des consultants] après tu feras ce que tu voudras avec... tu auras un stock de masques pour une quinzaine de jours ou quelque chose comme ça, chacun (...)

Inès [reprenant le cours de sa simulation où elle l'avait arrêtée] : moi je pose mon sac ici... [sur son poste de travail] je vais à mon casier... [déplace son avatar] je prends mon masque... je le mets... [l'animateur groupe le symbole "masque" avec le symbole "avatar"] je prends ma tasse... je vais à la cuisine [déplace son avatar masqué] je lance la machine à café, je prends mon café... voilà, et puis je vais à ma place [déplace son avatar] et j'y reste... (...)

Aude [a pris la main sur le pointeur] : donc moi je suis arrivée... on passe les détails de mon arrivée, mais grosso modo je suis venue d'ici, du couloir... [déplace son avatar dans le couloir] 
Animateur: alors tu croises quelqu'un dans le couloir... imaginons que Inès est repartie prendre un café [déplace l'avatar d'Inès dans le couloir et le met à côté de celui de Aude, révélant au passage la superposition des zones de distanciation physique intégrées aux avatars]

Sylvie : ben on peut pas se croiser là...

Nils : oui...

Jean-Christophe : en fait ça veut dire qu'il faut des masques... qu'il faut le masque sur tous les axes de circulation...

Plusieurs participants, ensemble : oui...

Animateur: mais je te rappelle, Inès, que pour aller chercher ton masque dans le casier tout à l'heure...

Inès : oui, $\mathrm{j}$ 'avais pas de masque... eh ben du coup, ça veut dire qu'il faut avoir un masque sur la tronche quand on arrive... (...) et il y a toujours un jour où on va arriver et on n'en aura pas dans le sac...

Animateur : du coup, imaginons une chose alors... on a un distributeur de masques à l'entrée... [place un symbole "masque" dans la zone d'entrée] quoi qu'il arrive tu viens en chercher là, avec un gel hydroalcoolique [un symbole "gel" se trouve déjà dans la zone d'entrée] et quand Inès elle arrive, ou n'importe qui qui arrive, tout de suite il a un masque... et si c'est un client, on lui propose aussi un masque...

Sylvie : ça je suis bien d'accord... (...)

Jean-Christophe: oui, donc ce que tu dis, c'est que ce n'est pas une dotation personnalisée en fait, ça n'a pas de sens... c'est plutôt un stock qui est mis à la disposition... voilà c'est ça... oui c'est plutôt pas mal, c'est plutôt cohérent parce qu'en fait on va tous avoir des situations différentes... »

La simulation révèle ici la diversité potentielle des situations que les usagers des locaux peuvent être amenés à rencontrer, mettant à l'épreuve les règles proposées dans la première version du protocole sanitaire (dotation individuelle de masque dans le casier personnel). L'intention louable de cette mesure fait cependant apparaître des failles dans la prévention (" ben on peut pas se croiser là... »). Le dispositif proposé permet alors d'envisager des alternatives, en favorisant l'élaboration collective de nouvelles règles (un stock de masques d'accès libre pour tous à l'entrée des locaux) et leur représentation sur le support (le positionnement d'un symbole «masque » dans la zone d'entrée). Un nouveau protocole, plus adapté aux logiques d'action des consultants, peut alors s'envisager. Le dispositif conçu s'avère donc servir le geste professionnel de l'intervenant, consistant à faire instruire le processus de prescription à l'aune de l'activité simulée.

\section{Un territoire d'intervention visant le développement à distance de "savoir-faire de présence "}

Dans le contexte pandémique, l'enjeu de prévention porté par la simulation du travail à distance s'avère dès lors particulièrement pertinent. En référence à la figure $1 \mathrm{a}$, on voit comment la simulation permet de redéfinir le protocole de prescription élaboré initialement, mais aussi de développer de nouvelles logiques d'action chez les consultants relevant de savoir-faire de prudence (Bourgeois \& Van Belleghem, 2004 ; $\mathrm{Cru}$, 2014). Rappelons-nous qu'à cette période (avril 2020), l'expérience que la population pouvait avoir des savoir-faire de protection sanitaire dans des lieux collectifs de vie ou de travail était quasi-nulle. Et le risque étant justement logé dans la co-présence, un dispositif de simulation en présentiel ne pouvait être proposé aux participants au risque de les mettre en danger. Pour le dire par une formule, c'est le développement de nouveaux « savoir-faire de présence » que le dispositif de simulation 
distancielle nourrit ici. Il permet ainsi de reconduire, selon une visée légitime, un geste professionnel traditionnel de l'ergonomie dans un environnement numérique et distanciel tout en restant fidèle aux principes conceptuels et opératoires de la discipline.

Le territoire d'intervention que balise l'intervenant est dès lors tout à fait singulier : il couvre le collectif d'acteurs qu'il ne peut réunir en présentiel, mais dont l'objet de l'interaction est justement d'expérimenter, par avance, des protocoles et des logiques d'action propres à l'interaction présentielle dans un espace donné. Prolongé auprès de différentes instances (mandataires sociaux, collectif de consultants) et ayant conduit à la rédaction d'un protocole sanitaire interne, ce travail a ainsi posé les jalons d'un territoire d'intervention articulant une facette physique (un dispositif socio-technique permettant à des consultants répartis sur le sol national d'interagir et de produire collectivement grâce à des ressources adaptées leur ayant été mises à disposition), une facette organisationnelle (constituée de consignes et règles d'échanges annoncées par avance et structurant le processus d'instruction du projet) et une facette existentielle (forte de la personnalité des consultants et prolongeant, malgré la période de crise, leur histoire commune).

Le dispositif technique expérimenté a été rapidement partagé auprès de la communauté des Ergonomes Solidaires, sous la forme d'un manuel pratique (Van Belleghem, 2020) visant une mise en œuvre simple et rapide pour accompagner les enjeux de prévention sur la période du déconfinement. Mais conçu sur une interface aux fonctionnalités restreintes (avatars manipulables par un seul participant à la fois) et un mode de représentation graphique basique (PowerPoint), le dispositif s'avérait limité pour accompagner des projets plus complexes. Accompagner un projet de coconception ambitieux et très participatif nécessitait, assurément, le développement d'un dispositif plus puissant. Une autre intervention allait en donner l'occasion.

\section{Nouveaux territoires de la conception : scénariser et simuler à distance le « travail à distance »}

\section{Une nouvelle université aux implantations territoriales multiples}

L'Université $\mathrm{U}$ est née du rapprochement récent de plusieurs établissements d'enseignement et de recherche aux profils variés (université, institut de recherche, écoles d'ingénieurs et d'architecture). À sa création, elle accueille près de 17000 étudiants et 500 doctorants. Si les établissements fondateurs sont situés principalement sur le même campus en région parisienne, l'un d'eux dispose aussi de campus répartis sur l'ensemble du territoire national (Lille, Nantes, Lyon, Marseille...). De fait, l'université nouvellement créée jouit d'une implantation nationale qui la démarque dans son offre de recherche et d'enseignement et qu'elle entend valoriser. Mais cette originalité territoriale doit aussi être gérée administrativement et techniquement.

Pour cela, l'université a souhaité bénéficier d'un accompagnement au changement centré sur les cultures, activités et pratiques de travail des personnels, de l'ordre de 3000 agents. L'enjeu était d'accompagner, dans le cadre de conduites de projets spécifiques, les processus de fusion des directions regroupées au sein de la Direction Générale des Services (agence comptable, direction financière, DRH, etc.). La direction 
des services informatiques (DSI) est de celles-là. Composée des anciens services informatiques des différents établissements fondateurs, regroupés provisoirement de façon "juxtaposée ", la nouvelle direction doit inventer son organisation future. Il s'agit de créer un nouvel ensemble garantissant la cohérence et le maintien en conditions opérationnelles de l'infrastructure et des systèmes informatiques pour l'Université U, mais aussi des services rendus à ses bénéficiaires (enseignants, chercheurs, étudiants, services administratifs, etc.), quelle que soit leur implantation sur le territoire national.

Une intervention est engagée en septembre 2020 pour réaliser l'accompagnement de ce projet $^{6}$. Elle vise, à travers une démarche de design participatif ambitieuse (Michel \& Haeffner, 2019), à faire co-concevoir des scénarios d'organisation possibles par les collaborateurs de la DSI avant de les évaluer par la simulation du travail, de façon à retenir les options les plus adaptées. La démarche envisagée est organisée en quatre cycles d'ateliers mobilisant chacun trois groupes de travail d'une quinzaine de personnes, eux-mêmes divisés en sous-groupes pour produire autant de propositions d'organisation. Ces groupes réunissent des personnels de la DSI, mais aussi des représentants de leurs bénéficiaires (responsables de services administratifs, enseignants, chercheurs, représentant des étudiants...). C'est ainsi près d'une cinquantaine de personnes représentatives de l'éco-système d'acteurs concernés qui est mobilisée sur la co-construction d'un schéma organisationnel cible devant répondre aux ambitions de la nouvelle université. L'outillage envisagé initialement pour la construction des scénarios d'organisation est particulièrement concret : grands formats de carton-plume, papiers colorés, ciseaux, post-it, feutres... L'enjeu est d'engager un processus d'idéation répondant aux exigences de représentation et de modification (cf. supra) permettant aux groupes de construire un support (une «maquette» de l'organisation-cible) sur lequel des simulations pourront ensuite être engagées.

Mais programmés à partir d'octobre 2020, ces travaux de groupe en présentiel vont être brutalement remis en cause par l'annonce du deuxième confinement, qui allait s'avérer long et drastique pour les universités. La direction, ne souhaitant pas reporter la réflexion en la conditionnant à la perspective d'un retour au présentiel incertain, exprime le souhait de poursuivre l'intervention en distanciel. Un dispositif de scénarisation et de simulation à distance devait être imaginé pour cela.

\section{La nécessité d'un outil de scénarisation puissant}

50 Les applications de visioconférence s'étant fortement améliorées et généralisées depuis le premier confinement, la perspective de faire interagir des groupes d'une quinzaine de personnes ne posait a priori pas de problème. Les fonctions permettant de diviser un groupe en plusieurs sous-groupes s'étant elles-mêmes démocratisées (surtout à l'université où les cours à distance s'appuient sur ce principe pour faire travailler les étudiants en sous-groupes au sein d'un cours commencé en plénière), l'engagement d'interactions à différentes échelles s'avérait tout à fait faisable. La difficulté résidait plus spécifiquement dans la mise à disposition d'un outillage favorisant le processus d'idéation pour "produire » collectivement des scénarios d'organisation. La fonction sur Teams permettant de « donner le contrôle » à un seul participant distant (cf. supra) était ici insuffisante pour soutenir ce processus de co-construction. Ce sont bien tous les participants qui devaient pouvoir agir en même temps sur un support unique, en donnant à voir leur action tout en visualisant les actions des autres. Enfin, cet outil 
devait pouvoir être pris en main rapidement sans formation préalable, excluant les outils complexes de conception collaborative utilisés, par exemple, en ingénierie ou en architecture. Une recherche des outils existants sur le marché a permis d'identifier l'application Miro comme répondant à ces critères.

Miro est une plateforme collaborative permettant à des équipes distantes de travailler ensemble sur un même «tableau blanc» («whiteboard») en ligne (Figure 4). Les pointeurs de souris des participants sont présents et visibles simultanément sur le «board " apparaissant à l'écran de chaque utilisateur, donnant à voir les productions de chacun. Utilisé en association avec la visioconférence, il permet des échanges oraux combinés à des actions sur le board, facilitant ainsi la co-production de représentations communes. Le board peut être zoomé et dézoomé sans limites, autorisant des productions à différents niveaux de détails, se complexifiant volontiers au fur et à mesure des travaux. Chacun pouvant se déplacer librement sur le board, des zones de travail peuvent être prédéfinies par les animateurs pour inviter les participants à venir produire, en sous-groupes sous l'outil de visio-conférence, leur propre scénario. Enfin, Miro dispose de nombreuses fonctionnalités graphiques (formes libres, flèches, logos, bibliothèques d'icônes et d'émojis, modèles divers - organigrammes, logigrammes, "cartes mentales", etc.), le rendant particulièrement adapté à la production de représentations à dominante graphique.

Figure 4 : À gauche : tableau blanc de l'outil Miro représentant quatre zones de scénarisation distinctes. À droite : le scénario en cours de construction du sous-groupe 2.

Figure 4 : Left : Miro tool whiteboard representing four distinct scenario areas. Right : scenario under construction of subgroup 2

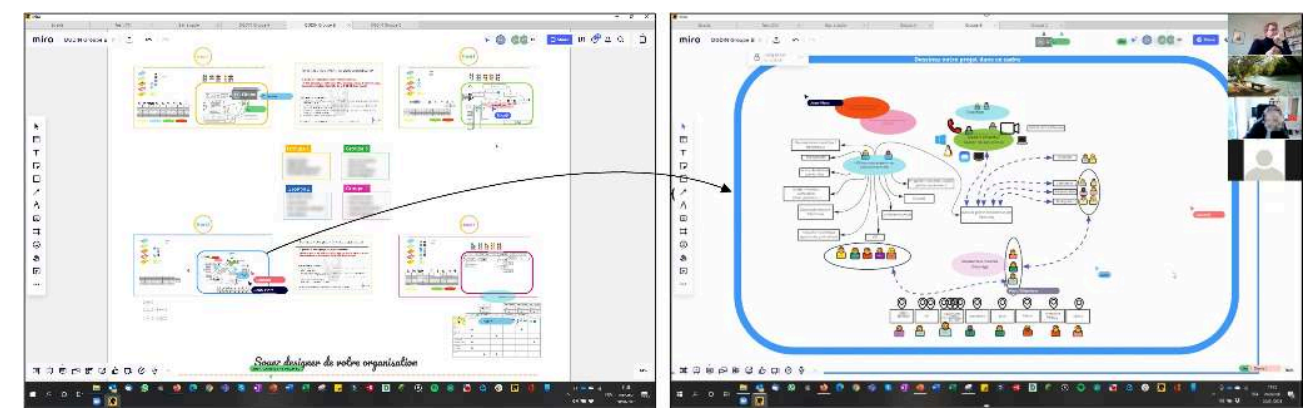

\section{Une activité de scénarisation et de simulation organisationnelle soutenue par l'outil collaboratif}

Les deuxième et troisième cycles d'ateliers ont été entièrement consacrés à la production de scénarios d'organisation sous Miro. Les fonctionnalités graphiques de l'outil collaboratif associées à l'usage de la visioconférence ont permis un travail d'idéation particulièrement créatif. Avec l'accord des participants, certains groupes ont pu faire l'objet d'un enregistrement sous Zoom pour garder la trace des processus collectifs de scénarios sous Miro. Là encore, un montage a posteriori a été réalisé pour rendre compte plus largement de ces processus auprès des différentes instances du projet. L'échange ci-dessous (cycle 2, groupe 3, sous-groupe 2) rend compte de la dynamique d'activité de co-conception articulant interactions verbales et manipulations d'objets partagés entre les participants du groupe (le scénario en cours de conception est représenté sur la figure 4 , à droite). 
Participant 1: «Et donc là, ce qu'on voulait faire, c'était relier les petits bonhommes de l'assistance à maîtrise d'ouvrage aux petits bonhommes... ben, qui sont là... bon, $\mathrm{y}$ en a peut-être trop des bonhommes là quand même [collègue acquiesce]... peut-être on aurait pu faire une petite bande de bonhommes, genre on les met tous là... [déplace et regroupe les icônes de personnages, avec l'aide de Participant 2]... je sais pas s'il en faut quatre ou... [aligne les icônes de personnages]... voilà, là on est bien, là....

Participant 2, acquiesçant : $\mathrm{mmh} \mathrm{mmh}$... [entoure les icônes de personnages d'un ovale blanc à contour noir] voilà... comme ça ?

Participant 1: Super! Voilà, comme ça, ça veut dire que c'est un groupe de personnes... et celles qui ont des couleurs...

Participant 2 : oui, c'est les entités...

Participant 1 : oui les entités... ou les fonctions essentielles qu'on peut imaginer

d'une DSI (...) voilà quoi...

Participant $2: \mathrm{mmh}$ mmh...

Participant $1:$ donc là il faut faire une flèche entre les deux... [désigne l'endroit avec son pointeur]

Participant 2 : oui... [relie les deux ovales par une double flèche]

Participant $1: .$. dans les deux sens, ça me semble pas mal... comme ça on voit un peu le rôle central de l'assistance à maîtrise d'ouvrage... »

L'analyse de cet échange et des actions associées montre que le dispositif proposé permet effectivement, à travers l'interface et ses fonctionnalités, une production graphique collaborative efficace ( ... peut-être on aurait pu faire une petite bande de bonhommes, genre on les met tous là... [déplace et regroupe les icônes de personnages, avec l'aide de Participant 2] »). Chaque participant peut agir simultanément sur l'objet produit en donnant à voir ses propres actions, mais aussi en venant compléter les actions des autres, en collaboration (par exemple lorsque Participant 1 émet des souhaits ( donc là il faut faire une flèche entre les deux... [désigne l'endroit avec son pointeur]») que Participant 2, plus à l'aise avec l'usage de l'outil, effectue immédiatement (« oui... [relie les deux ovales par une double flèche]). Le dispositif proposé permet ainsi la réalisation de scénarios d'organisation répondant aux deux premières exigences d'un support de simulation (représentation et modification collective du système de prescription en cours de conception) et permet de servir, avec efficacité, le geste professionnel de l'intervenant.

54 Les deuxième et troisième cycles d'ateliers ont permis de produire onze, puis sept scénarios différents. Leurs principes structurants ont été repris pour construire deux scénarios de synthèse volontairement opposés et les soumettre à la simulation lors d'un quatrième cycle d'ateliers. L'enjeu était de donner la possibilité d'évaluer, parmi une bibliothèque de situations rédigées sur des "post-its numériques » et présentées sur une zone du whiteboard (Figure 5, gauche), l'efficacité des logiques d'action collectives qu'invitait à mettre en œuvre chacun des deux scénarios. Là encore, les simulations sont enregistrées avec l'accord des participants et les séquences les plus significatives font l'objet d'un montage pour restitution ultérieure. L'extrait ci-dessous (cycle 4, groupe 2, sous-groupe 2) rend ainsi compte de la logique d'action retenue sur le scénario 2 (Figure 5, droite) pour répondre à une demande de l'Agence Comptable de bénéficier d'un développement informatique sur une application existante.

Animateur, zoomant sur la zone de simulation du scénario 2 de l'écran partagé :

« Donc scénario 2, voilà... alors dites-nous comment vous avez fait...

Participant 1: donc en fait l'utilisatrice, l'agent comptable (...), souhaite faire

évoluer son logiciel... (...) Elle fait une demande sur un ticket qui est flagué sur

l'outil de ticketing (...) donc là ce sera une demande d'évolution [désigne en la suivant 
avec son pointeur la flèche reliant la demande initiale à l'outil de ticketing]... donc dans le Pôle Appui SI Administration, l'équipe AMOA reçoit la demande [suit la flèche allant du ticketing à l'AMOA] et l'AMOA va communiquer... je peux peut-être faire un trait là... [trace une flèche entre l'AMOA et l'agente comptable]... des échanges avec le métier pour vraiment connaître leur besoin... et une fois que le besoin a été défini, le cahier des spécifications techniques, cahier des charges, etc. rédigés, on transfère à l'équipe développement de l'Appli métiers [...] d'où le lien là, AMOA, Appli métiers [désigne la flèche reliant les deux entités](...) ensuite, une fois que le développement est terminé (...) l'équipe développement doit informer l'équipe Support [suit la flèche la reliant] (...)

Animateur : parfait... bon ben c'est bien (...)

Participant 2 : et donc le moyen de communication entre l'AMOA et l'administratif [les désigne], ça peut être du mail, du chat, de la visio, du téléphone... tout moyen disponible... (...) le point d'entrée c'est le ticket, le point de sortie c'est le ticket, mais les échanges entre l'AMOA et le demandeur (...) peu importe le support... »

Figure 5 : À gauche : support de simulation comprenant la bibliothèque de situations et les différentes zones de simulations comparatives. À droite : simulation en cours sur le scénario 2 Figures 5 : Left : simulation support including the situation library and the different areas of comparative simulations. Right : scenario 2 simulation in progress

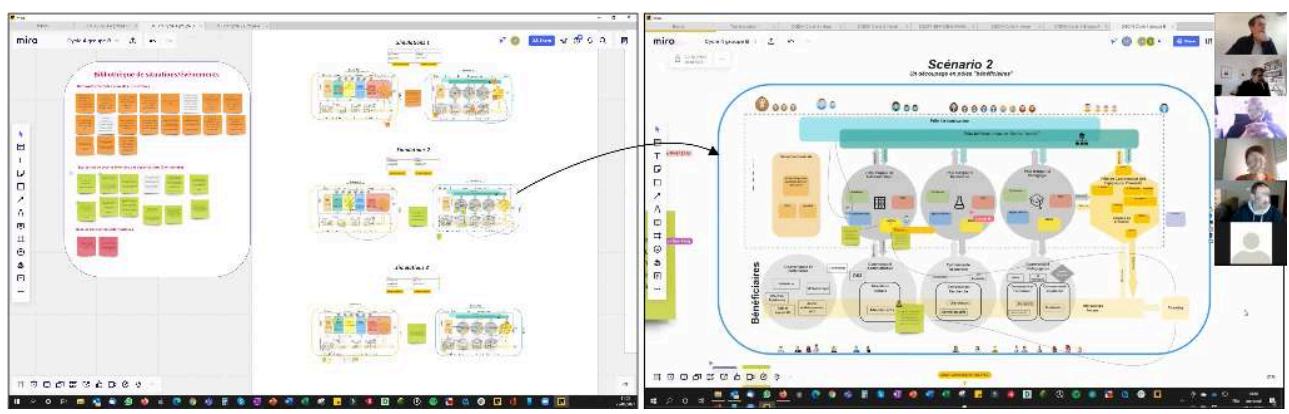

L'analyse de cet échange montre que les simulations portent moins ici sur la description incarnée d'activités individuelles (on n'y observe pas l'usage du «je») que sur les logiques de coordination et de coopération à savoir faire vivre entre les différents acteurs pour répondre au besoin exprimé. Cette observation est classique et pertinente en phase amont de l'avancement du projet, où les simulations visent plus volontiers la construction de logiques d'action collectives (matérialisées notamment par les multiples flèches entre acteurs) que des stratégies individuelles. C'est ici la boucle d'itération entre la simulation de l'activité et les scénarios de logiques d'action qui est à l'œuvre (cf. Figure 1a).

C'est aussi à ce moment que s'expriment des logiques d'un futur travail à distance, exploitant diverses possibilités d'interaction ( du mail, du chat, de la visio, du téléphone... tout moyen disponible... ») entre les acteurs de la DSI et les bénéficiaires, quelle que soit leur localisation géographique. La « simulation à distance » du travail semblait ouvrir la voie à la simulation "du travail à distance ", l'expérience de l'une rendant possible l'advenue de l'autre.

\section{Un territoire d'intervention favorisant une large participation au processus de co-conception}

Tout au long de la démarche, un lien de correspondance a commencé à se dessiner entre le caractère distanciel de l'intervention menée et le caractère territorial de la nouvelle DSI. En effet, les principes structurants émergeant des ateliers ont rapidement 
acté l'idée que le caractère multi-sites de l'organisation à construire pouvait être traité à travers un fonctionnement distanciel. L'expérience acquise du télétravail et de la visioconférence depuis le premier confinement permettait d'explorer des pistes où l'organisation serait décorrélée de l'implantation géographique grâce à la mise en œuvre de pratiques collaboratives à distance. Progressivement, le territoire du travail de la DSI s'est ainsi dessiné, articulant des activités de support aux utilisateurs directs et des activités de services aux bénéficiaires institutionnels de l'université pouvant s'exercer sous une diversité de modalités, présentielles ou distancielles. Le caractère distanciel de l'intervention, cité par les collaborateurs, semblait lui-même justifier ce choix.

Réalisée entièrement à distance (à l'exception de quelques rencontres et analyses en septembre 2020), l'intervention ergonomique avait en effet su baliser et couvrir un territoire tout à fait original. Quatre cycles de trois ateliers de $4 \mathrm{~h}$ chacun mobilisant près de cinquante participants répartis sur l'ensemble du territoire national dont beaucoup ne se connaissaient pas initialement : tel a été le dispositif mis en œuvre pour imaginer, concevoir et éprouver par la simulation la nouvelle organisation de la future DSI de l'Université. Encadrée par un dispositif soutenu d'arbitrage (Comité de pilotage) et de partage des résultats des ateliers (webconférence de l'ensemble des personnels), mis en œuvre lui aussi en distanciel, la dynamique mise en place a ainsi structuré un territoire d'intervention inédit, articulant une facette physique (constituée notamment de l'ensemble des sites et ressources informatiques réparties sur le sol national permettant la mise en lien du collectif d'acteurs et sur lequel s'est appuyé le dispositif technique de simulation), une facette organisationelle (structurée par les règles annoncées du vaste dispositif participatif proposé) et une facette existentielle (en capacité de faire se rencontrer et échanger les "cultures informatiques » des deux organisations que le diagnostic initial avait identifié comme enjeu majeur).

Mis en œuvre intégralement depuis le domicile des intervenants, le geste professionnel lié à la scénarisation et la simulation du travail a permis d'embarquer un nombre et une diversité d'acteurs rarement égalée dans une mise en œuvre en présentiel. Restait à savoir si ces gestes professionnels liés à la simulation distancielle pouvaient être enseignés... et là encore à distance.

\section{Nouveaux territoires de la formation : former à distance à la simulation à distance}

\section{Quelle continuité pédagogique pour les travaux pratiques?}

Le master d'ergonomie du CNAM des Pays de la Loire vise à former des ergonomes capables de mener une intervention ergonomique dans les champs du travail, de l'innovation et de la conception. Menée en alternance, la deuxième année est consacrée à l'accompagnement d'un projet d'entreprise. Les enseignements soutiennent cette intention. Deux unités d'enseignements portent plus spécifiquement sur la démarche ergonomique de conception de situations de travail. Depuis 2012-2013, la méthodologie de simulation du travail y est particulièrement développée et fait l'objet de plusieurs travaux pratiques (TP). L'un permet de dérouler, sur quatre séances, les grandes étapes de la méthodologie. Un autre permet d'expérimenter, sur trois séances, les spécificités de la simulation organisationnelle ${ }^{7}$. 
61 La rentrée de l'année 2020-2021, comme dans toutes les universités, s'est engagée dans un format présentiel guetté par la perspective d'un basculement rapide en distanciel. Celui-ci s'est confirmé le 30 octobre 2021, date du deuxième confinement national. L'enseignement a été maintenu dans le cadre d'un plan de continuité pédagogique mettant à disposition des enseignants, enseignantes, auditeurs et auditrices ${ }^{8}$ une plateforme d'outils numériques éprouvée de longue date au sein du CNAM et renforcée lors du premier confinement. Pour autant, l'enseignement à distance ne saurait se substituer intégralement à l'enseignement en présentiel. Les travaux pratiques, notamment, sont l'occasion d'un apprentissage centré moins sur l'acquisition de connaissances théoriques (qu'un cours magistral à distance permet d'assurer) que le développement par l'expérimentation de gestes professionnels spécifiques à la discipline enseignée. La dimension physique et corporelle de ces gestes professionnels est parfois grande, particulièrement quand ils visent l'usage, voire la fabrication, de ses propres outils. C'est le cas des travaux pratiques sur la simulation du travail, où les auditeurs sont invités à concevoir, construire, manipuler et faire manipuler les différents supports utiles à sa mise en œuvre. Formaliser des situations d'action caractéristiques, concevoir un scénario d'évènements, fabriquer une maquette, choisir un avatar en capacité d'incarner l'activité, préparer les scénarios de prescription à simuler, préparer l'animation de la séance de simulation, animer les simulations en groupe de travail (en jeu de rôles par exemple), faire évaluer la pertinence des scénarios simulés, conduire et formaliser les arbitrages en cours de simulation, etc. sont autant de savoir-faire professionnels qui ont intérêt à être éprouvés et évalués en formation pour être opérationnels dans le réel de l'intervention. Autrement dit, il s'agit de mettre en situation les auditeurs "d'avoir à faire ce qu'ils auront à faire » en intervention, notamment dans le cadre de leur alternance. C'est la capacité d'agir du futur intervenant que les travaux pratiques sur la simulation, à travers l'expérience de celle-ci, doivent ainsi contribuer à développer (Van Belleghem, 2018b). Le caractère présentiel du TP permet à la fois de répondre à la dimension physique de ces gestes professionnels et à la dimension collective de l'apprentissage (travaux de groupes).

L'enseignement à distance des travaux pratiques étant maintenu, il fallait tenir cette exigence de mise en situation, y compris en y intégrant le contexte probable des auditeurs, dont plusieurs devaient eux-mêmes réaliser leur stage en partie ou totalement à distance. Les inviter à développer des gestes professionnels en expérimentant des travaux pratiques à distance sur la simulation à distance leur permettant, possiblement, de mettre en œuvre une démarche de simulation à distance dans leur stage, devenait ainsi un objectif à la fois pertinent en termes d'enseignement et adapté à leur contexte d'intervention. Les quatre premières séances ont permis de proposer un enseignement descendant sur les principes de la simulation intégrant de nombreux cas d'intervention illustrés de séquences vidéo. Ces séquences constituent un premier apprentissage des logiques de mise en œuvre concrète de la méthodologie en situation. Les trois séquences suivantes ont été consacrées à définir les principes de la simulation distancielle à partir des premières interventions réalisées dans ce cadre (cf. chapitres précédents), à inviter les auditeurs à concevoir leur propre dispositif de simulation à distance et à l'animer collectivement dans une ultime séance. Le cas pratique était le même que celui utilisé les années précédentes pour le TP de simulation organisationnelle ${ }^{9}$, mais réalisé ici entièrement en distanciel. 


\section{Un support à inventer pour simuler l'activité temporelle d'un poste de nuit}

Le TP vise à concevoir un dispositif de simulation organisationnelle pour une entreprise fictive (bien qu'inspirée d'une intervention réelle) fabriquant industriellement des pizzas surgelées en $2 \times 8$. La problématique porte sur la création d'un poste de nuit d'une fonction particulière, le Conducteur-Trancheur (CT) pour assurer des tâches de nettoyage, de montage et de réglage de machines de tranchage (de chorizo et de pains de mozzarella) réparties sur deux lignes de fabrication. Une prescription d'aménagement des horaires de nuit du CT détaille le phasage temporel des tâches à réaliser, mais celles-ci doivent être réalisées en même temps que le nettoyage du secteur par des prestataires de service. Le TP invite les auditeurs à jouer le rôle des ergonomes engagés dans cette intervention et ayant proposé à la direction de vérifier, par la simulation, si la co-activité entre les différentes fonctions est possible tout au long de la nuit. L'enjeu est d'évaluer, voire de modifier, la conception des programmes horaires de l'une et l'autre des activités, détaillant le phasage des opérations prescrites à réaliser en coordination.

auditeurs, répartis en groupes de quatre ou cinq en visioconférence, doivent dans un premier temps concevoir le support de simulation. Ils sont invités à choisir l'outil qui doit leur permettre de répondre aux exigences attendues de celui-ci (représentation, modification, simulation). Plusieurs pistes leur sont proposées (ppt, sharepoint, Miro...), mais ils sont libres d'en suivre d'autres.

Une fois l'outil retenu, ils doivent imaginer et concevoir leur support de simulation. Partant d'une page blanche, ils sont invités à faire preuve de la plus grande créativité possible, à l'image de la situation que rencontrera l'ergonome professionnel qu'ils sont appelés à devenir. Le plus souvent, leur choix s'oriente vers une représentation de la dimension temporelle de l'activité sous forme de frise horaire, horizontale ou verticale, éventuellement complétée d'un plan du secteur de production. Les avatars retenus peuvent être autant des personnages à déplacer sur le plan que des éléments à déplacer le long de la frise temporelle, parfois même des machines à déplacer et à démonter ou des post-its « actions » à rédiger et à positionner temporellement.

La dernière séance du TP est consacrée à l'animation d'une simulation sur le support conçu. Tous les auditeurs sont réunis en séance plénière en visioconférence et un jeu de rôle est engagé. Le groupe présentant son support joue le rôle de l'équipe d'ergonomes conduisant l'intervention et devant animer, ici, la simulation. Les différents acteurs de l'entreprise (CT, prestataires, représentant de la direction, représentant du personnel, chef d'atelier) sont joués par des auditeurs des autres groupes. Les auditeurs jouant les $\mathrm{CT}$ et les prestataires se trouvent alors engagés dans une double projection: simuler l'activité d'opérateurs eux-mêmes en situation de simuler leur propre activité dans le projet.

Mais l'enjeu du TP réside moins dans la simulation des activités des opérateurs que dans la simulation de l'activité de l'ergonome professionnel animant un dispositif de simulation en entreprise. L'objectif pédagogique est ici de développer un geste professionnel d'ergonome à travers sa mise en situation réaliste. De ce point, il s'agit d'une simulation du travail à vocation formative (Pastré, 2005) inscrit dans le cadre d'un parcours d'apprentissage professionnel. L'originalité du dispositif tient ici dans l'objet de l'apprentissage par la simulation portant sur... la simulation. 
ette simulation de simulation (Van Belleghem, 2018b) revêt, en cet automne 2020, un caractère tout à fait particulier : elle prend la forme d'une simulation à distance de simulation à distance. L'exercice, pour déstabilisant qu'il puisse paraître au départ, n'en produit pas moins une mise en situation tout à fait pertinente, tant pour l'acquisition des gestes professionnels liés à la maîtrise de la simulation du travail que pour le développement de la maîtrise des outils d'interactions à distance, en passe de devenir des compagnons de route de ces auditeurs en master 2 engagés depuis la rentrée dans un stage en entreprise réalisé, pour la plupart d'entre eux, à distance.

\section{Une simulation (à distance) de simulation (à distance)}

69 Chaque mise en situation se déroule sur $30 \mathrm{mn}$. Afin de reproduire la configuration du groupe de travail d'entreprise, seuls les caméras et microphones des animateurs (jouant le groupe d'ergonomes) et des participants (jouant les acteurs de l'entreprise) sont allumés, les autres auditeurs étant en observation. Les séances sont enregistrées avec l'accord des auditeurs.

Le jeu de rôles reproduisant un groupe de travail réunissant les acteurs de l'entreprise en visioconférence, le groupe d'ergonomes doit se répartir les tâches et dérouler son animation comme il le ferait en réalité. La présentation du support, de son intention et de son fonctionnement est une première étape incontournable. D'elle dépend l'engagement efficace des simulations qui vont suivre. En soi, elle participe pleinement du geste professionnel à développer. Savoir réaliser ce geste professionnel à distance est aussi une compétence à acquérir. L'extrait ci-dessous, illustré par la figure 6, rend compte de sa mise en œuvre par un des groupes ayant retenu Miro comme outil interactif.

Aura $^{10}$ / rôle d'ergonome : « eh bien bonjour à tous... merci de nous avoir rejoints... Groupe de travail : bonjour... bonjour... bonjour...

Aura / ergonome: donc vous avez tous accès à un écran assez grand... [l'écran partagé rend visible tout le dispositif de simulation sur lequel les participants promènent leur pointeur de souris](...) l'objectif aujourd'hui c'est qu'il y a un planning qui a été envisagé sur la nouvelle organisation, notamment de la nuit [déplace le board sur le planning de nuit] et le but c'est de savoir si ce planning est réaliste et réalisable... et le deuxième objectif, c'est de savoir si géographiquement, sur la zone, il peut y avoir une co-activité possible entre l'équipe de nettoyage et le conducteur trancheur qui sera là de nuit (...)... donc je vais zoomer un petit peu... [zoome sur la zone de simulation] avec la roulette de votre souris vous pouvez approcher ou dézoomer... ici vous avez chacun un rôle, donc un avatar... un avatar peut être pris en cliquant dessus et en le déplaçant [saisit un avatar, le déplace puis le remet à sa place] et tout le monde va le voir se déplacer... donc ça, ça fait partie des éléments mobiles que vous pouvez prendre... (...) nous en parallèle, avec mon équipe, on va également mettre en place tout ce que vous faites sur le planning en bas [déplace le board pour rendre visible un tableau sous la zone de simulation] donc ça vous n'avez pas à le gérer, mais par contre on vous interrompra peut-être pour nous faire préciser combien de temps ça prend à peu près... (...) le but aujourd'hui c'est déjà de tester un scénario à peu près normal... donc on a plusieurs cartes situations [déplace le board vers la zone des cartes Situations] et aujourd'hui on va jouer celle-ci [saisit une carte et la place dans la zone de simulation] donc la carte Situation $\mathrm{n}^{\circ} 1$ : on est dans la zone de garnissage et les deux lignes ont été utilisées... et donc on va simuler à partir de l'arrivée des équipes... » 
Figure 6 : Structuration du dispositif de simulation proposé par un groupe d'auditeurs Figure 6 : Structuring of the simulation system proposed by a group of students

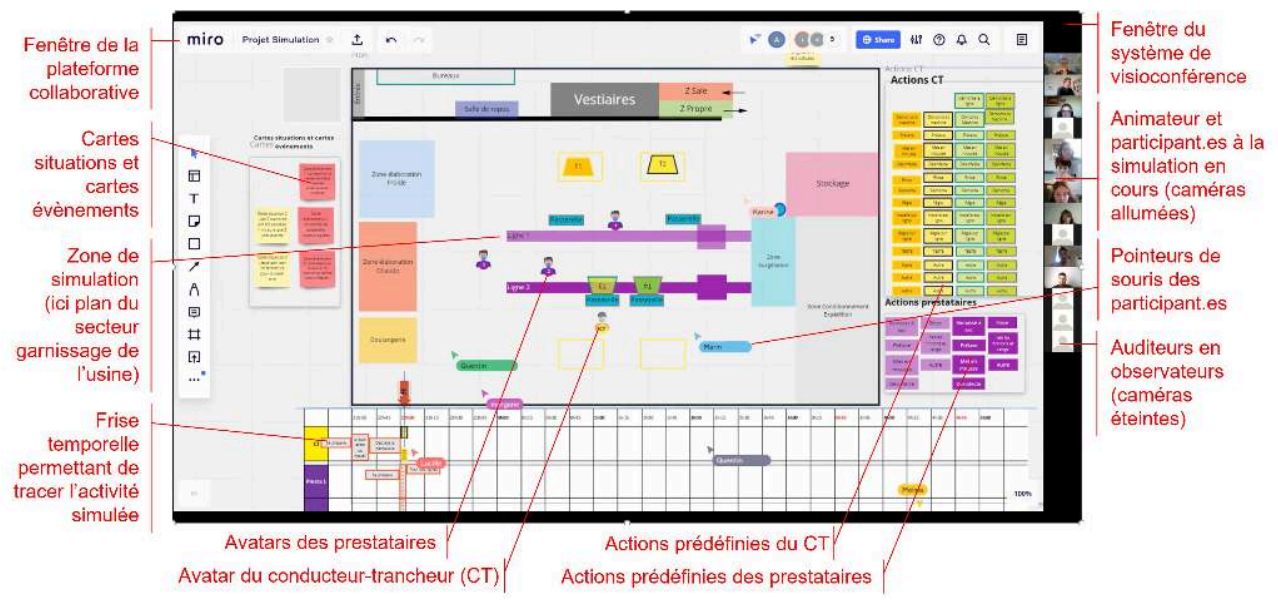

71 L'analyse de cet échange et des actions associées montre comment l'apprentie ergonome, acceptant le jeu de rôles dans lequel ses collègues se trouvent («eh bien bonjour à tous... merci de nous avoir rejoints... ») se projette très vite dans son propre rôle d'intervenante en donnant à découvrir le support de simulation, les modalités d'usage de l'interface (" avec la roulette de votre souris vous pouvez approcher ou dézoomer...»), la finalité de l'exercice (le but c'est de savoir si ce planning est réaliste et réalisable...), la fonction centrale des avatars ( ici vous avez chacun un rôle, donc un avatar... un avatar peut être pris en cliquant dessus et en le déplaçant [saisit un avatar, le déplace puis le remet à sa place] et tout le monde va le voir se déplacer... »), le rôle de l'équipe d'ergonomes ("nous en parallèle, avec mon équipe, on va également mettre en place tout ce que vous faites sur le planning en bas») et les situations à simuler, lui permettant de lancer les premières simulations («donc la carte Situation $n^{\circ} 1$ : on est dans la zone de garnissage et les deux lignes ont été utilisées... et donc on va simuler à partir de l'arrivée des équipes... "). L'acquisition du geste professionnel est engagée. La simulation commence.

Aura / rôle d'ergonome : "donc, ici en bas, sur notre frise chronologique, je vais mettre le curseur à $22 \mathrm{~h} \mathrm{30...} \mathrm{[déplace} \mathrm{le} \mathrm{curseur} \mathrm{de} \mathrm{la} \mathrm{frise} \mathrm{temporelle} \mathrm{sur} 22 \mathrm{~h} 30$ ] et donc à $22 \mathrm{~h} 30$ a priori, selon ce qui serait prévu, c'est que le conducteur-trancheur arrive... donc voilà je laisse peut-être la main au conducteur-trancheur, donc c'est Marin, de se saisir de son avatar, et de nous décrire, finalement, à partir de $22 \mathrm{~h} 30$ tout ce qu'il va faire dans ses activités...

Marin / rôle de conducteur-trancheur (CT) : ben déjà moi, j'arrive un peu avant $22 \mathrm{~h} 30$ pour être prêt... [saisit son avatar] j'arrive et je vais jusque dans les vestiaires pour mettre mes vêtements de travail [déplace son avatar dans les vestiaires]

Aura / ergonome: d'accord... [Quentin / Ergonome déplace l'action prédéfinie "se prépare" vers la frise, puis Lucille / Ergonome la dimensionne sur l'échelle de la frise temporelle à l'heure indiquée de l'activité simulée]

Marin / CT : je vais chercher mon équipement [déplace son avatar dans la zone de stockage] (...) une fois que j'ai pris mes outils, je me dirige vers les passerelles pour les démonter... [déplace son avatar vers les passerelles de la ligne 1] et donc je les mets sur les côtés... [déplace la première, puis la deuxième passerelle avec son avatar][Quentin et Lucille / Ergonomes tracent à nouveau l'activité simulée]

Aura / ergonome: donc ça pour démonter une passerelle, ça prend combien de temps?

Marin / CT : les passerelles, elles sont fixées au sol et après elles sont sur roulettes, donc il faut dévisser certains éléments... je dirais une bonne dizaine de minutes... [Lucille / Ergonome ajuste temporellement les actions de déplacement des passerelles] (...) 
Lucille / ergonome : là on arrive à peu près à $23 \mathrm{~h}$, les prestataires arrivent à ce moment-là...

Aura / ergonome : alors l'équipe de prestataires, comment ça se passe ? (...)

Karine / prestataires : euh... en général lorsqu'on arrive, nous actuellement, c'est déjà démonté... [positionne les trois avatars des prestataires sur les deux lignes] [Mélina / Ergonome trace les actions des prestataires sur la frise temporelle]

Aura / ergonome: alors... on a vu là, avec le curseur, quand on a avancé temporellement, que pour l'instant on était plutôt dans un état avec la ligne 1 qui était démontée et la ligne 2 qui n'était pas encore démontée (...)

Karine / prestataires : après nous on peut commencer par une ligne et aller sur la seconde quand elle est démontée... [déplace les avatars de prestataires vers la ligne 1] on peut effectivement modifier notre fonctionnement si ça nous fait pas perdre de temps...

Aura / ergonome: parce que du coup, j'ai une question, vous commencez systématiquement par la ligne 1 ?

Karine / prestataires: non, en fait on fait les deux en parallèle, sauf que là, si effectivement la 2 n'est pas prête, on peut très bien faire la 1 et ensuite aller sur la $2 \ldots$ »

L'analyse des échanges et des actions associées montre comment l'animatriceergonome s'astreint à inviter les participants à dérouler leur activité (et non uniquement leurs tâches) en saisissant leur avatar (" ... donc voilà je laisse peut-être la main au conducteur-trancheur, donc c'est Marin, de se saisir de son avatar, et de nous décrire, finalement, à partir de $22 \mathrm{~h} 30$ tout ce qu'il va faire dans ses activités... ") puis à guider, relancer, interroger l'activité en cours de déroulement. Dans ce cas de figure, la dimension temporelle de l'activité est particulièrement suivie au regard de la problématique posée («donc ça pour démonter une passerelle, ça prend combien de temps?»). Un partage des tâches entre ergonomes est prédéfini, certains animant la simulation tandis que d'autres prennent des notes et tracent l'activité et les problématiques rencontrées directement sur la frise temporelle du support, leur permettant de suivre le déroulement temporel et de relancer les simulations en conséquence ("là on arrive à peu près à $23 \mathrm{~h}$, les prestataires arrivent à ce moment-là... »). L'acquisition du geste professionnel se poursuit, soutenue par un dispositif technique ici particulièrement bien conçu.

Les simulations sont toutes suivies d'un débriefing avec l'ensemble des auditeurs, permettant d'évaluer la pertinence du support conçu, son efficacité à soutenir l'activité de simulation, son utilisabilité en situation, etc. C'est aussi l'occasion d'évaluer la dynamique d'animation mise en œuvre par l'équipe d'ergonomes. Ouvrir la séance et présenter la méthodologie et sa finalité, guider l'activité de simulation de l'activité des participants, faire évaluer les durées des actions réalisées pour les tracer, repérer les problématiques du travail rencontrées, proposer des pistes d'amélioration pour débloquer les difficultés éventuelles, faire explorer de nouvelles logiques d'action individuelles et collectives par les opérateurs, re-scénariser des options organisationnelles et/ou techniques, mobiliser les autres acteurs (direction, représentants $d u$ personnel...) pour discuter ou arbitrer des choix... sont autant d'exigences à tenir dans la mise en œuvre efficace du dispositif. Ce sont aussi autant de dimensions du geste professionnel qui trouvent l'occasion de se développer dans l'expérience de la simulation. 


\section{Un territoire d'intervention visant la construction à distance de l'expérience pratique}

Pour les enseignants, le territoire d'intervention est là encore tout à fait singulier. Agissant à distance des formés, il leur faut pour autant savoir (et pouvoir) engager un travail collaboratif orienté vers la construction de l'expérience pratique. Le transfert de connaissances théoriques doit laisser la place au développement d'une activité concrète, partagée entre les enseignants et les auditeurs, et dont la dimension tangible est centrale, fut-elle médiée par des outils numériques. Permettre de fabriquer, tester, essayer, manipuler, se tromper, recommencer, jouer... sont autant de caractéristiques de la mise en pratique à laquelle la formation doit inviter.

L'exercice est d'autant plus situé qu'il ouvre la perspective, pour les auditeurs, d'explorer un nouveau territoire d'intervention qu'ils pourront étendre eux-mêmes à leur entreprise s'ils devaient en être maintenus physiquement à distance. Le territoire d'intervention de l'enseignant se voit ainsi prolongé de la diversité des territoires d'intervention des apprenants qu'il leur permet d'ouvrir.

Mais au-delà de la mise en œuvre de ce TP, c'est ici le territoire de l'enseignement dans son ensemble qui a permis cet exercice, repérable à travers ses facettes physiques (constituée notamment de la palette d'outils mis en place de longue date par le CNAM dans une politique de service couvrant le territoire national et visant à donner accès largement à ses formations à une population majoritairement salariée et non forcément mobile), organisationnelle (par la mise en place d'un protocole de maintien des cours à distance, mais aussi du suivi des présences et d'évaluation des auditeurs sur les différents cursus pour assurer la continuité pédagogique) et existentielle (à travers notamment la mobilisation sans faille des personnels administratifs et des enseignants titulaires et vacataires dans un dispositif éprouvant, mais porteur des valeurs liées à la formation des adultes). Il y a là le jalonnement d'un territoire d'action collective qui a su, au moins temporairement, s'affranchir de la contingence géographique pour maintenir à l'œuvre sa vocation pédagogique à l'échelle du territoire national.

\section{Conclusion}

L'innovation que représentent les outils d'interaction à distance est ambivalente. Si elle permet d'ouvrir et d'explorer des territoires d'activité nouveaux, y compris sur les champs d'intervention traditionnels de l'ergonomie, elle ne saurait se substituer complètement à l'activité réalisée en présentiel. La visioconférence, même très outillée, laisse de côté des pans entiers de l'activité sociale et collaborative que la co-présence permet. On sait que l'espace, en tant qu'il est un médiateur de l'activité, soutient ces effets de collaboration et qu'il peut même être conçu spécifiquement suivant cet objectif (Heddad, 2016). Des écosystèmes de relations s'élaborent ainsi naturellement au sein des espaces physiques, de la salle de réunion au réseau de campus d'une université en passant par l'étage d'un service administratif, l'atelier de production d'une usine agroalimentaire, les implantations régionales d'une PME ou le réseau d'agences d'une banque internationale. À chacun de ces niveaux se tissent des liens de coopération structurant des formes d'agir productif commun et que les espaces physiques contribuent à tisser. De ce point de vue, la définition d'un territoire ne peut 
se passer totalement d'un ancrage géographique, tant la dimension spatiale contribue à la structuration des interactions des acteurs.

Pour autant, on ne saurait nier les perspectives qu'ouvrent les technologies d'interactions à distance et la diffusion des pratiques associées dans la façon dont les collectifs d'acteurs peuvent se recomposer et étendre leurs territoires d'activité. Les confinements successifs ont eu un effet d'accélérateur dont on peut croire qu'il laissera des traces durables dans les pratiques d'interactions à venir, tant professionnelles que privées. L'ergonomie ne se situe pas à l'écart de ces transformations. Ce qu'aura changé la crise sanitaire dans le travail changera nécessairement le rapport de l'ergonomie à celui-ci (au risque, sinon, qu'elle perde son rapport privilégié avec lui). Nos méthodologies comme nos approches théoriques doivent y être sensibles et savoir accompagner, voire devancer, ces transformations.

De ce point de vue, le regard rétrospectif porté dans cet article sur l'évolution de la simulation du travail doit pouvoir se lire selon trois axes.

Un axe historique d'abord: l'émergence et l'usage de la simulation distancielle dans le temps de l'événement pandémique nous rappellent que l'innovation nait de la contrainte. L'exploration des usages de la simulation distancielle durant une année, sur des interventions couvrant des domaines diversifiés de l'ergonomie (prévention, conception, formation) marque l'intérêt d'une méthodologie qui a permis de rester au service d'entreprises et d'organismes dans lesquelles une intervention présentielle n'était pas possible au moment où elle était requise. Le caractère historique de cette contrainte, avec ses rebondissements et ses déconvenues, de confinement en confinement, a guidé autant les techniques employées que les usages possibles de la simulation distancielle.

81 Un axe méthodologique ensuite: encore confidentielle, la simulation distancielle a intérêt à être partagée et mise en œuvre plus largement, à un moment où la fin de la pandémie n'est pas encore annoncée, et où l'on perçoit que le monde du travail d'après ne ressemblera pas au monde du travail d'avant la pandémie. La mise à distance du travail, à travers la généralisation du télétravail, les mobilités géographiques et les nouvelles pratiques collaboratives qu'elle a engendrées, ouvre à de nouveaux territoires du travail que nos méthodologies doivent savoir appréhender pour répondre à leurs besoins.

Un axe prospectif enfin : la simulation du travail est un geste professionnel important dans la palette professionnelle de l'ergonome, mais d'autres sont évidemment impactés par la mise à distance du travail, à commencer par l'observation ergonomique des situations d'activité. Comment accéder à l'activité lorsqu'elle se réalise majoritairement à distance? Quelle légitimité avons-nous, le cas échéant, à observer l'activité de travailleurs à leur domicile ou dans tout autre espace qu'ils auraient choisi d'investir ? Quels modèles de l'activité collective nous faut-il savoir construire pour rendre compte d'un agir productif commun réalisé intégralement par des individus isolés? Quelles méthodologies d'analyse à distance du travail à distance, par exemple à travers des observations de séances de travail en visioconférence, devons-nous développer ? Et enfin, de quel «terrain » parlons-nous, dans ce contexte, lorsqu'il s'agit de s'y rendre (et plus encore lorsqu'il s'agit d'inviter des étudiants à y aller eux-mêmes)? assurément pas refermé. La simulation du travail à distance est une réponse partielle, 
mais concrète, aux questions qui se posent actuellement à l'ergonomie. D'autres doivent être instruites. Gageons qu'elles permettront d'étendre encore nos territoires d'activité.

\section{BIBLIOGRAPHIE}

Andersen, S.N., \& Broberg, O. (2016). A framework of knowledge processes in participatory simulation of hospital work systems. Ergonomics, 60(4), 1-39. https://doi.org/ 10.1080/00140139.2016.1212999

ANI (2020). Accord national interprofessionnel du 26 novembre 2020 pour une mise en œuvre réussie du télétravail. https://medef-ain.fr/wp-content/uploads/2020/11/ANI-

T\%C3\%A91\%C3\%A9travail-26-11-2020.pdf

Asselineau, A., Albert-Cromarias, A., \& Ditter, J. (2014). L'écosystème local, ressource clé du développement d'une entreprise. Entreprendre \& Innover, 4(4), 59-70. https://doi.org/10.3917/ entin.023.0059

Besnard, L., \& Levy, E. (2017). Comment outiller une démarche de réflexion sur la gestion temporelle par la simulation managériale? [Communication] $52^{e}$ Congrès de la Société d'Ergonomie de Langue Française, Toulouse, France. https://ergonomie-self.org/document/comment-outillerune-demarche-de-reflexion-sur-la-gestion-temporelle-par-la-simulation-manageriale/

Blanc, Y. (2020). Valeur et territoire. [Conférence] Séminaire du pôle ergonomie de l'université Paris 1, 25-27 mai 2020, Paris, France.

Bobillier Chaumon, M.-E., Rouat, S., Laneyrie, E., \& Cuvillier, B. (2018). De l'activité DE simulation à l'activité EN simulation : simuler pour stimuler. Activités [en ligne]. https://doi.org/10.4000/ activites.3136

Boudra, L. (2016). Durabilité du travail et prévention en adhérence : le cas de la dimension territoriale des déchets dans l'activité de tri des emballages ménagers [Thèse de doctorat, Lyon II]. Theses.fr. http:// www.theses.fr/2016LYSE2171\#

Boudra, L., \& Béguin, P. (2013). Quels liens entre territoire et travail ? Le cas des centres de tri des déchets d'emballages ménagers [Conférence]. $48^{e}$ congrès international de la SELF, Paris. https:// ergonomie-self.org/document/quels-liens-entre-travail-et-territoire-le-cas-des-centres-de-trides-dechets-demballages-menagers/

Bourgeois, F., \& Van Belleghem, L. (2004). Avec l'approche travail dans l'évaluation des risques professionnels (décret du 5/11/01), enfin du nouveau en prévention. In F. Hubault (Ed.), Travailler, une expérience quotidienne du risque? (pp. 147-165). Toulouse : Octarès.

Braatz, D., Paravizo, E., \& Fontes A.R.M. (2019). Simulation, Prototyping and Experimentation The Potential of the Maker Labs to Achieving a Design-Driven HFE. In S. Bagnara, R. Tartaglia, S. Albolino, T. Alexander, \& Y. Fujita (Eds.), Proceedings of the $20^{\text {th }}$ Congress of the International Ergonomics Association (IEA 2018). IEA 2018. Advances in Intelligent Systems and Computing, vol. 824. Springer, Cham. https://doi.org/10.1007/978-3-319-96071-5_205 
Casse, C. (2015) Concevoir un dispositif de retour d'expérience intégrant l'activité réflexive collective : un enjeu de sécurité dans les tunnels routiers [Thèse de doctorat, Université Grenoble Alpes]. Theses.fr. http://theses.fr/2015GREAH024

Conceição, C., \& Broberg, O. (2019). Simulation in Diagnosing and Redesigning Knowledge Transfer Systems in the Offshore Oil Industry. In S. Bagnara, R. Tartaglia, S. Albolino,

T. Alexander, \& Y. Fujita (Eds.), Proceedings of the $20^{\text {th }}$ Congress of the International Ergonomics Association (IEA 2018). IEA 2018. Advances in Intelligent Systems and Computing, vol. 821. Springer, Cham. https://doi.org/10.1007/978-3-319-96080-7_55

Cheyrouze, M. (2021). La conception du travail en horaires atypiques : une démarche d'innovation sociale conduite en milieu hospitalier [Thèse de doctorat, Toulouse 2]. Theses.fr. http://www.theses.fr/ s170048

Cru, D. (2014). Le risque et la règle. Le cas du bâtiment et des travaux publics. Toulouse : Éditions Érès.

Demissy, R. (2018). Trajectoires sectorielles longues et actions collectives territoriales : quelles capacités d'intervention pour les acteurs locaux ? Étude à partir de trois secteurs en Champagne-Ardenne [Thèse de doctorat, Sorbonne Paris Cité]. Theses.fr. http://theses.fr/2018USPCC045

Di Méo, G. (1998). Géographie sociale et territoires. Paris : Nathan.

Du Tertre, C. (2012). Lean production et modèles de valeur. Activités, (9)2. https://doi.org/ $10.4000 /$ activites.462

Gal-Petitfaux, N., \& Roche, L. (2015) Expériences corporelles et gestes professionnels. Recherches \& Éducations.

Haradji, Y. (2021). Simulation multi-agent de l'activité humaine : une concrétisation en ergonomie du programme de recherche technologique « cours d'action ». Activités, 18(1). http:// journals.openedition.org/activites/6166 ; https://doi.org/10.4000/activites.6166

Heddad, N. (2016) L'espace de l'activité : une construction conjointe de l'activité et de l'espace. Le Travail Humain, 80(2), 207-233.

Heddad, N. (2019). Contributions of Simulation for Developing New Activities: Simultaneous Approach of Activity and Space in the Case of a Fire Department Situation Room Design Process. In S. Bagnara, R. Tartaglia, S. Albolino, T. Alexander, \& Y. Fujita (Eds.), Proceedings of the $20^{\text {th }}$ Congress of the International Ergonomics Association (IEA 2018). IEA 2018. Advances in Intelligent Systems and Computing, vol. 821. Springer, Cham. https://doi.org/10.1007/978-3-319-96080-7_76

Le Berre, M. (1992). Territoires. In A. Bailly, R. Ferras, \& D. Pumain (Eds.), Encyclopédie de géographie (pp. 617-638). Paris : Economica.

Mazzola, V. \& Allain, D. (2020). Un atelier à distance pour l'aménagement de situations de travail. [Conférence] Séminaire du pôle ergonomie de l'université Paris 1, 25-27 mai 2020, Paris, France.

Mesnard, I. (2003) La réhabilitation des logements sociaux à travers les processus d'appropriation de l'espace par les habitants [Thèse de doctorat, CNRS]. Theses.fr. http://www.theses.fr/2003LYO20019

Michel, J.-C., \& Haeffner, I. (2019). La simulation du travail comme soutien à l'arbitrage dans une démarche de co-construction. [Conférence] Communication présentée au $54^{e}$ congrès de la SELF, Tours, France.

Pastré, P. (2005). Apprendre par la simulation. De l'analyse du travail aux apprentissages professionnels. Toulouse : Octarès. 
Ughetto, P. (2018). Simuler pour changer la conception et les rapports sociaux de conception du travail : un commentaire sur les dispositifs de simulation de l'activité dans les organisations. Activités, 15(1). https://doi.org/10.4000/activites.3101

Van Belleghem, L. (2012). Simulation organisationnelle : innovation ergonomique pour innovation sociale. In M.F. Dessaigne, V. Pueyo, \& P. Béguin (Eds.), Innovation et travail. Sens et valeurs du changement. Actes $d u 47^{e}$ congrès de la Société d'Ergonomie de Langue Française du 5, 6 et 7 septembre 2012. Lyon : Éditions du Gerra.

Van Belleghem, L. (2018a). La simulation de l'activité en conception ergonomique : acquis et perspectives. Activités, 15(1). https://doi.org/10.4000/activites.3129

Van Belleghem, L. (2018b) Faire l'expérience de l'intervention par la simulation. Éducation Permanente, 2018(1), 161-170.

Van Belleghem, L. (2020, 5 mai). Dispositif de simulation du travail à distance pour accompagner le déconfinement [Document PowerPoint].

Vermersch, P. (1994). L'entretien d'explicitation. Issy-Les-Moulineaux : ESF.

Vispi N., Lebrun Y., Lepreux S., Chaabane S., \& Kolski C. (2021). Simulation on RFID Interactive Tabletop of Working Conditions in Industry 4.0. In T. Borangiu, D. Trentesaux, P. Leitão, O. Cardin, \& S. Lamouri (Eds.), Service Oriented, Holonic and Multi-Agent Manufacturing Systems for Industry of the Future. SOHOMA 2020. Studies in Computational Intelligence, vol. 952. Springer, Cham. https://doi.org/10.1007/978-3-030-69373-2_24

Zittel, B. (2020). L'initiative du réseau Ergonomes Solidaires. [Conférence] Séminaire du pôle ergonomie de l'université Paris 1, 25-27 mai 2020, Paris, France.

\section{NOTES}

1. Cette approche n'est pas si éloignée de l'approche spatiale défendue par Heddad (2016), qui distingue d'un côté l'espace, traditionnellement entendu comme un lieu défini par ses caractéristiques matérielles et bâtimentaires, et de l'autre l'espace de l'activité, c'est-à-dire l'espace modelé et agi dans et par l'activité de travail.

2. On notera ici le contraste avec les jeux en ligne de nos enfants qui offrent des possibilités d'interactions à plusieurs dizaines, voire centaines, de participants particulièrement impressionnantes.

3. Plein Sens et l'auteur, à travers sa structure Realwork, sont associés. Ils partagent les mêmes locaux et interviennent régulièrement ensemble pour des clients communs mais aussi, comme c'est le cas ici, pour des problématiques internes.

4. Le dispositif a été conçu et animé par Laurent Van Belleghem (Realwork / Plein Sens), JeanChristophe Michel (Plein Sens) et Inès Haeffner (Plein Sens).

5. Les prénoms sont utilisés avec l'accord des personnes concernées.

6. L'intervention a été réalisée par Jean-Christophe Michel (Plein Sens) et Laurent Van Belleghem (Realwork / Plein Sens).

7. Le master est placé sous la responsabilité pédagogique de Christophe Real (CNAM Pays de la Loire). Les unités d'enseignement ERG220 et ERG226 sont sous la responsabilité de Flore Barcellini (CNAM Paris). Les travaux pratiques dont il est question ici sont assurés par Laurent Van Belleghem (Realwork) et Marie-Haude Guerry (Athanalys).

8. Au CNAM, les apprenants et apprenantes sont nommé.es auditeurs et auditrices.

9. Une description de ce TP et des résultats qu'il produit en mode présentiel a été détaillée par Van Belleghem (2018b). 
10. Les prénoms sont utilisés avec l'accord des personnes concernées.

\section{RÉSUMÉS}

La crise sanitaire qui s'est déclarée en 2020, par les contraintes qu'elle a fait peser sur les entreprises et la société, a généré des formes de mise à distance du travail (confinement, télétravail, jauges d'occupation des locaux, etc.). La continuité de l'activité économique s'est réalisée, dans de nombreux secteurs, par le recours massif aux technologies numériques, notamment de visioconférence. Ces modalités nouvelles ont reconfiguré les communautés de travail exerçant traditionnellement en présentiel. Ce sont dès lors les territoires du travail, c'est-àdire les écosystèmes de relations structurant l'agir productif commun, qui se sont vu profondément restructurés. Les ergonomes n'ont pas échappé à ces bouleversements, leurs territoires d'intervention se voyant bousculés autant que les territoires du travail des bénéficiaires de leurs prestations se sont transformés. Cet article se propose de rendre compte comment de nouveaux territoires d'intervention de l'ergonomie ont pu être explorés par l'application de ce geste professionnel qu'est celui de la simulation du travail dans un contexte où il ne pouvait être mené, comme à l'habitude, de façon présentielle. Après avoir précisé les notions de territoires d'intervention et rappelé les exigences méthodologiques de la simulation du travail, trois interventions engagées sur l'année 2020 dans le contexte pandémique sont détaillées. Elles rendent compte de la naissance et de la consolidation de la simulation du travail à distance, que l'on peut aussi nommer simulation distancielle. Ces interventions ont permis d'investir, malgré la distance, trois domaines privilégiés de l'ergonomie : le conseil en prévention, la conception de situations de travail et la formation.

Due to the constraints it has placed on businesses and society, the health crisis that broke out in 2020 has generated various forms of distancing from the workplace (confinement, teleworking, space occupancy gauges, etc.). In many sectors the continuity of economic activity has been achieved through the massive use of digital technologies, particularly videoconferencing. These new methods have reconfigured the work communities that traditionally practice face-to-face. It is therefore the territories of work, i.e. the ecosystems of relations that structure common productive action, which have been profoundly restructured. Ergonomists have not escaped these upheavals. Their territories of intervention have been shaken up to the same extent that the work territories of the beneficiaries of their services have been transformed. This article aims to report how new territories of intervention in ergonomics have been explored through the application of a professional gesture - the simulation of work - in a context where it could not be performed face-to-face. After clarifying the concepts of territory of intervention and recalling the methodological requirements of work simulation, we describe three interventions initiated in 2020 in the context of the pandemic. They reflect the birth and consolidation of remote simulation of work. Despite the distance, these interventions made it possible to examine three privileged areas of ergonomics: prevention advice, the design of work situations and training. 
INDEX

Mots-clés : ergonomie, conception, simulation de l'activité, simulation du travail à distance, simulation distancielle

Keywords : ergonomics, design, activity simulation, remote simulation

\section{AUTEUR}

\section{LAURENT VAN BELLEGHEM}

Ergonome consultant, REALWORK, 5, rue Jules Vallès, 75011 Paris.

laurent.vanbelleghem@realwork.fr 